\title{
Non-linear MHD modelling of Edge Localized Modes dynamics in KSTAR.
}

\author{
${ }^{1}$ M.Bécoulet, ${ }^{2}$ M. Kim, ${ }^{3}$ G.Yun, ${ }^{4}$ S. Pamela, ${ }^{1}$ J.Morales, ${ }^{1}$ X.Garbet, ${ }^{1,5}$ G.T.A.Huijsmans,
} ${ }^{1}$ C.Passeron, ${ }^{1}$ O.Février, ${ }^{7}$ M.Hoelzl, ${ }^{7}$ A.Lessig, ${ }^{7}$ F. Orain

${ }^{1}$ CEA, IRFM, 13108 Saint-Paul-Lez-Durance, France

${ }^{2}$ Ulsan National Institute of Science and Technology (UNIST), 44919 , Ulsan, Rep. of Korea

${ }^{3}$ Pohang University of Science and Technology, Pohang, Gyeongbuk 790-784, Rep. of Korea

${ }^{4}$ CCFE, Culham Science Centre, Oxon, OX14 3DB, UK Abingdon, UK

${ }^{5}$ Eindhoven University of Technology, Eindhoven, The Netherlands

${ }^{6}$ Max Planck Institute for Plasma Physics, Boltzmannstr. 2, 85748 Garching, Germany

\begin{abstract}
The explanation of the existence of the rotating MHD modes in the pedestal region before Type I Edge Localized Mode (ELM) crash and in the inter-ELM periods (ELM precursors) observed in KSTAR is provided for the first time in the present paper. The dynamics of ELMs, observed using Electron Cyclotron Emission Imaging (ECEI) in KSTAR tokamak, is compared to the modelling results of the non-linear reduced resistive MHD code JOREK. The realistic KSTAR pulse parameters and geometry including X-point and Scrape Off Layer (SOL) were used. The full ELM crash modelling was performed using JOREK code for single and multi-harmonic representation and in multi-cycles ELMy regimes including relevant flows. The most unstable toroidal modes numbers $(n=5-8)$, velocity ( $\sim 5 \mathrm{~km} / \mathrm{s}$ for $n=8$ mode) and the direction of the mode rotation were reproduced in modelling. The two fluid diamagnetic effects and toroidal rotations included in the model were found to be the most important factors in explaining the experimentally observed rotation of the ballooning modes before the ELM crash and in the inter-ELM phase. In multi-harmonic multi-cycle simulations the spectrum of temperature fluctuations is similar to the experimental one in the inter-ELM phase, where several rotating modes with medium $n$ numbers were detected in $5-30 \mathrm{kHz}$ frequency range. The rotating modes can contain single or several harmonics which last from $0.2 \mathrm{~ms}$ to few $\mathrm{ms}$ in time, and can appear and disappear in the inter ELM period or persist until a new ELM crash.
\end{abstract}

I.Introduction. The understanding of Edge Localized Modes (ELMs) physics is of particular importance for ITER where heat and particles fluxes due to ELMs represent a concern for the plasma facing components (PFC) [1]. The direct comparison of theory and modelling with experimental observations of ELM dynamics plays an important role not only in the theoretical understanding of ELMs. They are necessary for the reliable predictions of divertor heat and particle fluxes and for understanding of ELMs mitigation technics physics needed for their optimization in different ITER scenarios. The measurements performed with Electron Cyclotron Emission Imaging (ECEI) diagnostic on KSTAR [2,3,4] provided new surprising features of the Type I ELMy regimes on which the present paper is mainly focused. The 
rotating structures with medium toroidal mode numbers $(n=5-8)$ lasting about few milliseconds in time were detected in the pedestal region inside the separatrix during the interELM period and prior to the ELM crash (so called "precursor" phase). The observed modes rotate with frequencies of the order of the diamagnetic one $(5-20 \mathrm{kHz})$. Moreover a rapid change from a dominant mode number to another one or co-existence of few modes in the temperature fluctuation spectrum were detected on KSTAR using ECEI [4]. The direction of modes rotation on KSTAR can be both the electron and ion diamagnetic one [2-4]. Similar observations were done on AUG [5], MAST [6], NSTX [7], however the ELM precursors rotation was mainly observed in the electron diamagnetic direction. Linear MHD modelling for KSTAR discharge parameters [3] suggested that the ballooning/peeling modes destabilized in the pedestal are good candidates to explain the ECEI diagnostic observations, however only the linear stage was modelled and modes rotation was not explained in reference [3]. The observed regular rotation of the modes decreases while approaching ELM crash, then ELM filaments cross the separatrix [2,7]. During the ELM crash the expelled ELM filaments propagate in SOL and "blobs" are cut from the main plasma [8,9]. The understanding of full ELMs dynamics with self-consistent modelling of relevant flows is crucial in predictions of energy and particle losses during an ELM (ELM size) and even more importantly the structure and magnitude of transient heat and particle fluxes into the divertor during an ELM which remains an issue for ITER. In particular toroidal plasma rotation, but mostly the electron diamagnetic, ion diamagnetic and electrostatic drifts which are large in the steep pedestal gradients region influence ballooning modes stability [9], modes dynamics both on linear and non-linear (crash) phase [8,9] and divertor heat fluxes paten which is strongly influenced by drifts in confined plasma and in SOL $[10,15]$. The work presented in this paper is done as a part of the large ongoing research program aiming the understanding of MHD instabilities and their active control in tokamaks based on the first principles non-linear 
MHD modelling using JOREK code [8-10]. In the present paper the full dynamics of ELMs is studied in the multi-harmonics and multi-cycles regime which is shown to be a minimum model to capture the essential physics pushing modelling capabilities to the more realistic description of Type I ELMy H-modes. In our previous work [9] which was the first work on the subject of rotating ELM precursors it was shown that, before the ELM crash, the two fluid diamagnetic and electrostatic $\vec{E} \times \vec{B}$ drifts produce poloidal rotation of the ballooning modes in the range of diamagnetic frequencies, mainly in electron diamagnetic direction [9], similar to most experimental observations [2-7]. However, only one single harmonic was used and no direct comparison with experiment was done in [9]. In the present work, we modelled ELM precursors and filaments dynamics for the realistic KSTAR pulse \#7328 parameters $[3,4]$ and compared to the ECEI observations. In particular it was demonstrated that, in multiharmonics multi-cycle non-linear modelling including two fluid diamagnetic effects and toroidal rotation, many experimental observations can be reproduced, including localization of the modes, velocity and direction of the poloidal rotation and temperature fluctuations spectrum. The paper is organized as follows. In Sec.II, the JOREK model with flows and coordinate system is introduced. In Sec.III, the modelling results of full ELM crash due to a single ballooning mode $n=8$ for KSTAR parameters are presented. The poloidal velocity of the mode $n=8$ obtained in JOREK modelling is compared to the ECEI measurements of temperature fluctuations on KSTAR and theoretical predictions from [9]. The relaxation of density and temperature profiles, filaments dynamics and generation of poloidal mean flow [8,9] on the non-linear stage are described. In Sec. IV, the modelling of multi-harmonics $(n=1-8)$ and multi-cycles ELMs is presented. Temperature fluctuations spectrum in interELM regime showed similarities with experimentally observed one.

II. Model. In the present work, we used the non-linear resistive MHD code JOREK [8], with flows relevant for this study included in the model [10]. For the following discussion of the 
direct comparison between modelling and experiment here we recall the essential features which should be included in the model. In particular toroidal plasma rotation and two fluid diamagnetic effects are essential to describe the ballooning modes rotation in the pedestal region with a strong pressure gradient [9]. The magnetic field in JOREK code is represented in the form: $\vec{B}=F_{0} \nabla \varphi+\nabla \psi \times \nabla \varphi$, where $\varphi$ is toroidal angle, $\psi$-is the poloidal magnetic flux, and $F_{0}=B_{\varphi, 0} R_{0}, B_{\varphi, 0}$ being the toroidal field on the magnetic axis and $R_{0}$-is major radius. The plasma fluid velocity is taken in the following form:

$$
\begin{aligned}
\vec{V}_{\text {tot }} & =\vec{V}_{\|}+\vec{V}_{E \times B}+\vec{V}_{i}^{*} \approx \\
& \approx V_{\|} \vec{B}-\left(R^{2} / F_{0}\right)(\nabla u \times \nabla \varphi)-\delta^{*}\left(R^{2} / \rho\right)\left(\nabla p_{i} \times \nabla \varphi\right)
\end{aligned}
$$

Here the first term $\vec{V}_{\|}=\frac{\vec{B}}{B^{2}}\left(\vec{V}_{i}, \vec{B}\right)=V_{\|} \vec{B}$ - is the velocity parallel to the magnetic field. The second term is $\vec{E} \times \vec{B}$ convection where the electric field is expressed as gradient of the electrostatic potential: $\vec{E}=-\nabla u$. The last term is the ion diamagnetic drift velocity, where the parameter: $\delta^{*}=1 /\left(R_{0} \Omega_{c i}\right), \Omega_{c i}=\frac{Z_{e f f} e B_{0}}{m_{i}}$ is the ion gyrofrequency, $p_{i}$ - is ion pressure, $e=1.6022 \cdot 10_{S I}^{-19}$-is the elementary charge, $\rho=m_{i} n_{i}$-is the mass density. For simplicity in following the effective ion charge is taken $Z_{\text {eff }}=1 ; p_{i}=p_{e}=p / 2 ; T_{i}=T_{e}=T / 2$. The normalized set of reduced MHD equations with two fluid diamagnetic and neoclassical effects solved in the present work is similar to [10]:

$$
\begin{aligned}
& \frac{1}{R^{2}} \frac{\partial \psi}{\partial t}=\eta \nabla \cdot\left(\frac{1}{R^{2}} \nabla_{\perp} \psi-j_{A}\right)-\frac{1}{R}[u, \psi]-\frac{F_{0}}{R^{2}} \partial_{\varphi} u+\frac{\tau_{I C}}{\rho}\left(\frac{F_{0}}{R^{2}} \partial_{\varphi} p+\frac{1}{R}[p, \psi]\right) \\
& \nabla \varphi \cdot \nabla \times\left\{\begin{array}{l}
\rho\left(\frac{\partial}{\partial t}+\vec{V}_{\text {tot }} \cdot \nabla\right) \vec{V}_{E}+\nabla(\rho T)+ \\
+\nabla \cdot \Pi_{i}^{\text {neo }}-\vec{J} \times \vec{B}+\vec{S}_{V}-\vec{V}_{\text {tot }} S_{p}-v_{\perp} \nabla^{2} \vec{V}_{\text {tot }}
\end{array}\right\}=0 \\
& B\left\{\left(\frac{\partial}{\partial t}+\vec{V}_{\text {tot }} \cdot \nabla\right) \vec{V}_{\|}+\nabla(\rho T)+\nabla \cdot \prod_{i}^{\text {neo }}+\vec{S}_{V}-\vec{V}_{\text {tot }} S_{p}-v_{\|} \nabla^{2} \vec{V}_{\text {tot }}\right\}=0
\end{aligned}
$$




$$
\begin{aligned}
& \frac{\partial(\rho T)}{\partial t}=-\left(\vec{V}_{E}+\vec{V}_{\|, i}\right) \cdot \nabla(\rho T)-\gamma \rho T \nabla \cdot\left(\vec{V}_{E}+\vec{V}_{\|, i}\right)+\nabla \cdot\left(\mathrm{K}_{\perp} \nabla_{\perp} T+\mathrm{K}_{\|} \nabla_{\|} T\right)+(1-\gamma) S_{T}+0.5 V_{\text {tot }}{ }^{2} S_{p}(5) \\
& \frac{\partial \rho}{\partial t}=-\nabla \cdot\left(\rho \vec{V}_{\text {tot }}\right)+\nabla \cdot\left(D_{\perp} \nabla_{\perp} \rho\right)+S_{\rho}
\end{aligned}
$$

The normalization used here was the following: $\rho=\rho_{S I} / \rho_{0, S I}$, where $\rho_{0}=A Z_{e f f} m_{p} n_{e 0}$ is the mass density on the magnetic axis, $A=2$ for deuterium plasma, $t=t_{S I} / \sqrt{\mu_{0} \rho_{0}}, \mu_{0}=4 \pi 10^{-7}$, A Spitzer-like resistivity $\eta=\eta_{S I, 0} \sqrt{\rho_{0} / \mu_{0}}=\eta_{0}\left(T_{e} / T_{e, 0}\right)^{-3 / 2}$ is used, where $T_{e, 0}$ is the electron temperature at the magnetic axis. A temperature-dependent perpendicular viscosity is used: $v_{\perp}=v_{\perp, S I} \sqrt{\rho_{0} / \mu_{0}}=v_{\perp 0}\left(T_{e} / T_{e, 0}\right)^{-3 / 2}$, parallel viscosity was constant in modelling $v_{\|}=v_{\|, S I} \sqrt{\rho_{0} / \mu_{0}}$. The Braginskii parallel thermal conductivity is expressed as $K_{\|}=K_{\|, S I} \sqrt{\rho_{0} / \mu_{0}}=K_{\|, 0}\left(T_{e} / T_{e, 0}\right)^{5 / 2}$ The ratio of specific heat is $\gamma=5 / 3$. Perpendicular diffusion ad-hoc coefficients depends on radial coordinate (normalized magnetic flux: $\left.\psi_{n}=\left(\psi-\psi_{\text {axis }}\right) /\left(\psi_{\text {sep }}-\psi_{\text {axis }}\right)\right)$ : and are decreased to the neoclassical value in the pedestal region to represent the $\mathrm{H}$-mode transport barrier $D_{\perp}\left(\psi_{n}\right)=D_{\perp, S I}\left(\psi_{n}\right) \sqrt{\mu_{0} \rho_{0}}$, (in the present modelling we set perpendicular particle diffusion coefficient as follows : $D_{a x i s, \perp, S I} \sim 2 \mathrm{~m}^{2} / \mathrm{s}$, $\left.D_{p e d, \perp, S I} \sim 0.02 \mathrm{~m}^{2} / \mathrm{s}, \quad D_{S O L, \perp, S I} \sim 12 \mathrm{~m}^{2} / \mathrm{s}\right)$, the same profile for the perpendicular heat diffusion coefficient $K_{\perp}(\psi)=K_{\perp, S I} \sqrt{\mu_{0} \rho_{0}}$, with $K_{\|, 0} / K_{a x i s, \perp}=10^{8}$. The total plasma pressure is: $\quad p=\mu_{0}\left(p_{e}+p_{i}\right)_{S I}=\mu_{0} p_{S I}, \quad$ where $\quad p=\rho T, \quad T=\mu_{0} \rho_{0}\left(e / m_{i}\right) T_{e V}$-temperature, $u=u_{S I} \sqrt{\mu_{0} \rho_{0}} / F_{0}$-electrostatic potential. The sources of toroidal current $-j_{A}$, particles $-S_{p}$, and heating $-S_{T}$ have been introduced. A toroidal rotation source was introduced in the equation for the parallel velocity to maintain the rotation profile compensating losses due to the parallel viscosity: $S_{V}=-v_{\|} \Delta V_{\|, t=0}$. The toroidal current is: $J^{(3)} \equiv(\vec{J}, \nabla \varphi)=-j /\left(\mu_{0} R^{2}\right)$, 
where $j \equiv \Delta^{*} \psi=\frac{\partial^{2} \psi}{\partial^{2} Z}+R \frac{\partial}{\partial R}\left(\frac{1}{R} \frac{\partial \psi}{\partial R}\right)$. The neoclassical poloidal friction was taken in the simplified form similar to [10]:

$$
\nabla \cdot \Pi_{i}^{\text {neo }}=\mu_{i, \text { nеo }} \rho\left(B^{2} / B_{\theta}^{2}\right)\left(V_{\theta}-V_{\theta, \text { nеo }}\right) \vec{e}_{\theta}
$$

Introducing poloidal unit vector as: $\vec{e}_{\theta}=(R /|\nabla \psi|) \nabla \psi \times \nabla \varphi$, we obtain: $B_{\theta}=|\nabla \psi| / R$. The neoclassical poloidal velocity is expressed as follows: $V_{\theta \text {, neo }}=k_{i} \tau_{I C}\left(\nabla_{\perp} \psi \cdot \nabla_{\perp} T\right) / B_{\theta}$ and total poloidal velocity is $: V_{\theta}=\left[-\left(\nabla_{\perp} \psi, \nabla_{\perp} u\right)-\tau_{I C}\left(\nabla_{\perp} \psi, \nabla_{\perp} p\right) / \rho+V_{\|} B_{\theta}^{2}\right] / B_{\theta}$, where we used the normalization: $\quad V_{\theta}=V_{\theta, S I} / \sqrt{\mu_{0} \rho_{0}}, \quad \mu_{\text {neo,i }}=\mu_{\text {neo, }, \text { SI }} \sqrt{\mu_{0} / \rho_{0}}, \quad \vec{V}_{\|, S I} \sqrt{\mu_{0} \rho_{0}}=V_{\|} \vec{B}, \quad$ and $\tau_{\text {IC }}=m_{i} /\left(2 \cdot e \cdot F_{0} \sqrt{\mu_{0} \rho_{0}}\right) \cdot \tau_{\text {IC }} \approx 3.10^{-3}$ for KSTAR parameters used here. Typical values estimated in $[10,11]$ of the normalized neoclassical coefficients $\left(\mu_{i, \text { neo }}=2.10^{-5} ; k_{i}=-1.1\right)$ were taken constant in this modeling for simplicity. The normalized poloidal and parallel viscosities were set to: $v_{\|}=10^{-5} ; v_{\perp, 0}=10^{-6}$. Normalized central resistivity was $\eta_{0}=5.10^{-7}$ which corresponds to $\eta_{0, S I} \approx 1.510^{-6}(\mathrm{Ohm} \mathrm{m})$. Here, for numerical reasons, resistivity is 150 times larger than the experimental one: $\eta_{0, \text { exp }, S I}=5.2410^{-5} Z \ln \Lambda / T_{e[e V]}^{3 / 2} \approx 10^{-8}(\mathrm{Ohm} \mathrm{m})$ for central electron temperature $T_{e}(0) \sim 2 k e V$. The isoparametric cubic Bezier finite elements were used to construct 2D grid in the poloidal cross-section [12]. The continuity of all variables and their derivatives including coordinates $(\mathrm{R}, \mathrm{Z})$ is satisfied on this $\mathrm{C} 1$ grid. The finite element grid is aligned to equilibrium flux surfaces for the three regions of the core, the SOL and the private region. The boundary of the computational domain is limited by the flux surfaces and divertor target plates. The boundary conditions around the computational domain correspond to those of an ideally conducting wall, where all perturbations are set to zero. On the divertor targets Bohm sheath boundary conditions were used for the fluid velocity and the heat flux normal to the target plates: 


$$
\begin{aligned}
& \left(\vec{V}_{\text {tot }}, \vec{n}\right)= \pm C_{s} \\
& \left(n_{e} T \vec{V}_{\text {tot }}+K_{\|} \nabla_{\|} T\right) \cdot \vec{n}=\gamma_{s h} n_{e} T \vec{V}_{t o t} \cdot \vec{n}
\end{aligned}
$$

where $C_{s}=\sqrt{\gamma T}$ is the ion sound speed, $\gamma_{s h}$ is the sheath transmission factor (here we used $\gamma_{s h}=4.5$ ) and $\vec{n}$ is the unit vector normal to the divertor plate. The temperature and density have free outflow boundary conditions at the target. The toroidal dimension is represented by a Fourier series. The time stepping is done using the implicit Crank-Nicolson scheme $[8,12]$. Resulting sparse system of equations is solved using a Generalized Minimal REsidual Solver (GMRES) with the preconditioner obtained by solving independently each sub-matrix corresponding to non-coupled Fourier harmonics. These sub-matrices are solved using the direct parallel sparse matrix solver PaStiX [13]. First the simulation starts on the initial fluxaligned mesh only for axisymmetric $n=0$ component without other toroidal modes and continues until all flows (parallel and perpendicular) in the plasma core and SOL are established. Typical time to reach equilibrium with flows is about $\sim 0.5-1 \mathrm{~ms}$. Then other harmonics are initialized at the noise level. The JOREK code uses a left handed cylindrical coordinate system $(R, Z, \varphi)$. However for the practical reasons of the direct comparison of ECEI diagnoistic observbations the following analysis of the rotation of the ballooning modes, let us introduce more practical left-handed coordinate $\operatorname{system}\left(\vec{e}_{r}, \vec{e}_{\theta}, \vec{e}_{\varphi}\right)$, where $\vec{e}_{r}=\frac{\nabla \psi}{|\nabla \psi|} ; \vec{e}_{\theta}=\frac{R}{|\nabla \psi|} \nabla \psi \times \nabla \varphi ; \vec{e}_{\varphi}=\frac{\nabla \varphi}{R}$ (Fig.1). The magnetic field in the JOREK model is expressed as following: $\vec{B}=F_{0} \nabla \varphi+\nabla \psi \times \nabla \varphi$, so in equilibrium: $B_{r}=0 ; B_{\theta}=\frac{|\nabla \psi|}{R} ; B_{\varphi}=\frac{F_{0}}{R}$. According to the experimental setup [4] the direction of the toroidal velocity and the toroidal plasma current are the same (negative in our coordinate system $\left.\left(\vec{e}_{r}, \vec{e}_{\theta}, \vec{e}_{\varphi}\right)\right)$ corresponding to the co-NBI injection and opposite to the magnetic field which is positive in our system. Since the direction of the pressure gradient in equilibrium is 
always toward the plasma center, the direction of the ion diamagnetic drift velocity $\vec{V}_{i}^{*}=-\frac{\nabla p_{i} \times \vec{B}}{e n_{i} B^{2}} \approx-R^{2} \frac{\nabla p_{i} \times \nabla \varphi}{F_{0} e n_{i}}$ is clockwise (positive in our system) and for the electron diamagnetic drift velocity: $\vec{V}_{e}^{*} \approx R^{2} \frac{\nabla p_{e} \times \nabla \varphi}{F_{0} e n_{e}}$ it is anti-clockwise (negative in our system) (see Fig.1). The radial force-balance can be written as follows:

$$
\left(e n_{e}(\vec{E}+\vec{V} \times \vec{B})-\nabla p_{i}\right) \vec{e}_{r}=0
$$

In the $\operatorname{system}\left(\vec{e}_{r}, \vec{e}_{\theta}, \vec{e}_{\varphi}\right)$ :

$$
E_{r}=\frac{1}{e n_{e}} \frac{\partial p_{i}}{\partial r}+B_{\varphi} V_{\theta}-B_{\theta} V_{\varphi}
$$

Where in the derivation of (10) we used the following expressions:

$$
\begin{aligned}
& \left(\vec{E}+\vec{V} \times\left(F_{0} \nabla \varphi+\nabla \psi \times \nabla \varphi\right)-\nabla p_{i}\right) \frac{\nabla \psi}{|\nabla \psi|}=0 \\
& \frac{(-\nabla u, \nabla \psi)}{|\nabla \psi|}+F_{0} \frac{\nabla \psi(\vec{V} \times \nabla \varphi)}{|\nabla \psi|}+\frac{\nabla \psi}{|\nabla \psi|}(\vec{V} \times \nabla \psi \times \nabla \varphi)-\frac{\left(\nabla p_{i}, \nabla \psi\right)}{e n_{e}|\nabla \psi|}=0 \\
& E_{r}=\frac{(-\nabla u, \nabla \psi)}{|\nabla \psi|} ; \frac{\partial p_{i}}{\partial r}=\frac{\left(\nabla p_{i}, \nabla \psi\right)}{|\nabla \psi|} ; F_{0} \frac{\nabla \psi(\vec{V} \times \nabla \varphi)}{|\nabla \psi|}=-\frac{F_{0}}{R} \vec{V} \frac{R(\nabla \psi \times \nabla \varphi)}{|\nabla \psi|}=-B_{\varphi}\left(\vec{V}, \vec{e}_{\theta}\right) ; \\
& \frac{\nabla \psi}{|\nabla \psi|}(\vec{V} \times \nabla \psi \times \nabla \varphi)=\frac{\nabla \psi}{|\nabla \psi|}(\nabla \psi(\vec{V}, \nabla \varphi)-\nabla \varphi(\vec{V}, \nabla \psi))=\frac{|\nabla \psi|}{R} V_{\varphi}=B_{\theta} V_{\varphi}
\end{aligned}
$$

Note that in the pedestal region with rather steep pressure gradient typical for H-mode plasma, the radial electric field is usually negative (so called radial electric field "well" in the pedestal) when the first diamagnetic term in (10) is dominant. However at strong toroidal plasma rotation ( $V_{\varphi}<0$ in our reference frame) the radial electric field is increased and even can change sign to a positive one and, as it will be demonstrated later, it is the case for the KSTAR pulse we modelled in the present work. In earlier work [9], we showed that two fluid diamagnetic effects are essential for the explanation of the regular rotation of ballooning modes in the linear phase. In [9] scalar variables $(\psi ; p ; u)$ were decomposed in Fourier 
series. For example the magnetic flux is represented as : $\psi(r, \theta, \varphi, t)=\sum_{m, n} \psi_{m n}(r) e^{-i(m \theta-n \varphi+\omega t)}$. Note that here the convention is : $m=n q\left(r_{\text {res }}\right) m>0 ; n>0$. In the frame of reference rotating poloidaly with the velocity $V_{E \times B}=E_{r} / B_{\varphi}$ introduced in [9] , the equilibrium radial electric field was zero and only poloidal diamagnetic velocity for ions and electrons $V_{i, e}^{*}$ were considered in derivation of the dispersion relation for the ballooning modes in two approximations: high and low resistivity plasmas [9]. As it was shown in [9], in this reference frame all perturbations due to the unstable ballooning mode $(m, n)$ resonant at $q\left(r_{\text {res }}\right)=m / n$ do not rotate in highly resistive plasma $\Omega_{\theta \text {, mode }}^{f} \approx 0$. However the ballooning modes rotate poloidaly in low resistivity plasma with angular frequency: $\Omega_{\theta \text {,mode }}^{f} \approx 0.5 m \Omega_{i}^{*}$, where $\Omega_{i}^{*}=V_{i}^{*} / r, V_{i}^{*} \approx-\frac{1}{B_{\varphi} e n_{e}} \frac{\partial p_{i}}{\partial r}$, as it was predicted by ideal MHD theory with diamagnetic effects taken into account [14]. It was shown in [9] that for typical tokamak plasma parameters the approximation of ideal MHD is valid for most cases. In the non-rotating laboratory frame the poloidal velocity of the rotating frame $\left(V_{E \times B}\right)$ should be added to the poloidal mode velocity in the rotating frame $V_{\theta \text {,mode }}^{f}$ and hence:

$$
V_{\theta \text {,mode }, \text { lab }}=V_{\theta \text {,mode }}^{f}+V_{E \times B}
$$

Multiplying (11) by $\left(\mathrm{m} / \mathrm{r}_{\text {res }}\right)$ and using (10) the angular rotation frequency of the mode $(m, n)$ in the non-rotating laboratory frame $\left(\vec{e}_{r}, \vec{e}_{\theta}, \vec{e}_{\varphi}\right)$ (Fig.1) can be expressed as follows:

$$
\Omega_{\text {mode }, l a b}=m \Omega_{\theta, E \times B}+\Omega_{\theta, \text { mode }}^{f}=m\left(-\Omega_{i}^{*}+\Omega_{\theta, p l}\right)+\Omega_{\theta, \text { mode }}^{f}-n \Omega_{\varphi, p l}
$$

Where we used that: $\Omega_{\theta, E \times B} \approx V_{E \times B} / r_{r e s}=E_{r} /\left(B_{\varphi} r_{r e s}\right) \approx-\Omega_{i}^{*}+\Omega_{\theta, p l}-\Omega_{\varphi, p l} / q_{r e s}$, $q_{\text {res }} \approx B_{\varphi} r_{\text {res }} /\left(B_{\theta} R\right)=m / n, \Omega_{\theta, p l}=V_{\theta} / r_{\text {res }}, \Omega_{\varphi, p l}=V_{\varphi} / R$. And $\Omega_{\theta \text {,mode }}^{f} \approx 0.5 m \Omega_{i}^{*}$ - for ideal or low resistivity plasmas. And $\Omega_{\theta \text {, mode }}^{f} \approx 0$ for high resistivity plasma. Later on we will show 
that the expressions (11-12) obtained for a circular plasma remains also rather good approximation for the realistic non-circular plasma.

III. Single harmonic simulation. The initial equilibrium for the KSTAR pulse \#7328, $B_{\text {tor }}=2.25 T, I_{p}=750 \mathrm{kA}, q_{95}=5$ [3] was calculated by the EFIT code (EQDSK file) at the time $t=4.36 \mathrm{~s}$. Then the pedestal density and temperature profiles were modified and taken as it is shown in Fig.2. After that the magnetic equilibrium was recalculated self-consistently using the JOREK code and the flux aligned grid was constructed. Precise measurements of the pedestal profiles are not available on KSTAR so here we used the same procedure as in [3] which consisted in adjusting the pedestal pressure profile in a way to obtain the largest linear growth rate for the $n=8$ ballooning mode which was observed experimentally at the time considered here. Note that maximum pedestal pressure in Fig.2 is similar to one used in [3]. In the present work the bootstrap current is calculated using temperature and density profiles presented in Fig.2. The toroidal rotation profile was taken close to the experimental one measured by the charge exchange spectroscopy (CES) diagnostic (Fig.3). Then the equilibrium with flows was obtained at approximately $t=0.5 \mathrm{~ms}$ after running the JOREK code only for one harmonic $n=0$. In the KSTAR pulse \#7328 at time $t=4.36$ s (corresponding to the phase between ELM crashes or inter-ELM phase) the ECEI diagnostic localized on the Low Field Side (LFS) detected structures just inside separatrix with the main toroidal number $n=8$ which were rotating poloidaly with a velocity of about $V_{\text {mode, }, a b, \text { exp }} \approx 5.4 \mathrm{~km} / \mathrm{s}$ (Fig.4). After the equilibrium with flows was reached, a single harmonic $n=8$ was initialized in modelling with a small amplitude at the numerical noise level $\left(\sim 10^{-27}\right)$. Both linear phase and highly non-linear phases of ELM due to the ballooning mode $n=8$ including profiles relaxation were modelled. The time evolution of the magnetic energy of the single harmonic $n=8$ calculated by JOREK is presented in Fig.5. The temperature fluctuations for the $n=8$ ballooning mode inside separatrix in the frame corresponding to the ECEI observation window are presented in 
Fig.6. Here (a) and (b) images taken just before ELM crash are separated by 0.008ms (two points in time indicated by diamonds in Fig.5). Note that mode rotates poloidaly in the ion diamagnetic direction (clockwise direction) at about $\sim 5 \mathrm{~km} / \mathrm{s}$ which is similar to the experimentally observed $\sim 5.4 \mathrm{~km} / \mathrm{s}$. However as we discussed in Sec. II at slower plasma toroidal rotation typically the radial electric field is negative in the pedestal region. In this case the ballooning mode perturbations rotates in the electron diamagnetic (anti-clockwise in our frame) direction in the laboratory frame which is the most common observation in many tokamaks [2-7]. To verify this statement we did a run with the same form of the toroidal velocity profile but reduced central value: $V_{\varphi, 0}=54 \mathrm{~km} / \mathrm{s}$ instead of the experimental one: $V_{\varphi, 0}=325 \mathrm{~km} / \mathrm{s}$. As expected in the linear phase the mode $n=8$ was found to rotate poloidally in the electron diamagnetic direction at $\sim-7 \mathrm{~km} / \mathrm{s}$ for the case $V_{\varphi, 0}=54 \mathrm{~km} / \mathrm{s}$ (Fig.7). The radial profiles of the poloidal velocities $V_{\theta \text {,mode }}^{f} ; V_{\theta \text {,mode }, a b} ; V_{E \times B}$ over a line in the mid-plane $(Z=0)$ are shown in Fig.8 for these two cases. Note that the direction of the poloidal rotation of the mode $n=8$ changes from the ion diamagnetic direction (positive in Fig.8) at large experimental-like toroidal rotation $(325 \mathrm{~km} / \mathrm{s})$, to the electron diamagnetic direction (negative) at lower toroidal rotation $(54 \mathrm{~km} / \mathrm{s})$. In the non-linear phase of an ELM the magnetic perturbation is strong enough to force magnetic reconnections in the resistive plasma leading to the plasma edge ergodisation [8]. The magnetic topology near the X-point during an ELM crash at the time corresponding to the maximum of the magnetic energy of the $n=8$ mode (indicated by a star on Fig.5) is presented in Fig.9. The density filaments expelled from the main plasma at this time are presented in Fig.10. This modelling picture is very similar to experimental observations [2-4]. The snapshots of the temperature perturbations before and just after the ELM crash separated in time by $\sim 0.0166 m s$ are presented in Fig.11, where the ECEI diagnostic window at LFS mid-plane is indicated by the vertical lines. Note the dramatic change in the mode rotation after the frame (4) in Fig.11. This time corresponds to the 
maximum of the magnetic energy for the mode $n=8$ where actually the ELM crash starts. Approaching the crash the regular rotation of the mode first decreases and then becomes irregular and can change the direction and the amplitude in the narrow layers within the pedestal. The dynamics of ELM filaments at this stage is mainly defined by a strongly sheared mean poloidal flow which is generated due to the non-linear mode coupling via Maxwell stress tensor [8,9]. The poloidal plasma velocity profile during an ELM is presented in Fig.12. Note the strongly sheared poloidal flow in the pedestal region (Fig.13) leading to the deformation of the expelled filaments which are finally cut from the main plasma forming "blobs” (Fig.14). On the non-linear phase of an ELM the density and temperature profiles are relaxed (Fig.15). Note that in spite of the ballooning structure of the mode and its initial localization on the LFS, more ELM power is deposited into the inner divertor (in/out 2:1) (Fig.10) which is a typical feature observed in previous modelling including drifts [15] and in many experiments [16] pointing out on the importance of the drifts in the model to reproduce correctly power deposition in the divertor.

Sec. IV. Multi-harmonics multi-cycles ELMs modelling. The single harmonic modelling described above in Sec.III showed that if the ballooning mode is unstable, its structure, localization in the pedestal and poloidal rotation velocity are very similar to the ECEI observations in the pedestal region on KSTAR. However not all rotating ELM "precursors” observed in the experiment lead to ELM crash and typically several coherent modes lasting few $m s$ are observed at the same time in temperature fluctuation spectrum in the inter-ELM periods [4], which cannot be explained in single harmonic and single ELM simulation presented above. To consider more realistic conditions multi-mode and multi-ELM cycles were studied in this work. In particular multi-cycles regime is important for a reason that in the typical single ELM modelling the all modes are initialized at the low amplitude (at computer noise level $\sim 10^{-27}$ ) and initial pressure profile is already unstable. However after the 
first crash and pressure profile relaxation the residual multi-modes MHD turbulence remains on a detectable level compatible to the measured by ECEI. Moreover the second ELM will appear in modelling only when the pedestal pressure gradient is sufficiently large to destabilize ballooning modes and the following ELMs can be due to the different most unstable n-numbers compared to the first ELM limiting the pressure gradient in selfconsistent way compared to the first ELM crash which starts from the already unstable initial pressure profile [15]. As it was shown in [15] the two fluid diamagnetic effects are essential to stabilize the residual after crash MHD permitting the pedestal to be rebuilt again provided the existence of the heat and particle sources which should be strong enough to compensate and overcome the transport due to the residual MHD turbulence. Based on these findings we propose here the generic picture of the possible explanation of the ballooning modes behavior before ELM crash (rotating precursors) and existence of the rotating multi-modes in the interELM periods detected by ECEI diagnostics. Fist note that during an ELM crash the multiple ballooning modes can be unstable linearly or due to non-linear coupling $[15,17]$, hence multiple n-s modes can exist after a crash. Secondly while the pedestal pressure gradient is increasing again the diamagnetic stabilization becomes stronger (the $3^{\text {rd }}$ term in equation (1)) and some modes and especially for n-numbers [15] can be totally stabilized and hence disappear from the spectrogram. On the other hand other ballooning modes can become unstable when corresponding critical pressure gradient is reached. As a consequence new multiple rotating ballooning modes can be destabilized and detected like rotating structures inside separatrix or lines on the temperature fluctuations spectrogram. They can last for a certain time and not necessarily immediately lead to the large ELM crash since they produce continuous small transport delaying pedestal rebuilt by sources. These is a 'competition' between the transport out of the pedestal due to the weakly unstable ballooning modes and heat and particle sources. Finally the next ELM crash can happen due to the most unstable 
mode when pressure gradient is large enough. The most unstable mode number may be different from the previous ELM. Firstly we concentrated on the non-linear ballooning modes coupling and the residual multi-modes MHD turbulence remaining after the first ELM crash which correspond to the inter-ELM period. In Fig.16 the magnetic energies of $n=1-8$ modes are presented for the JOREK run for the same plasma parameters (Fig.2-3), but in multiharmonics regime. One can see that for the first ELM the $n=8$ mode remains the most unstable and other modes $n=4-7$ are also linearly unstable, but with smaller growth rates than $n=8$ (Fig.16). Approaching the non-linear phase all modes became strongly coupled. Note in particular that $n=1-3$ modes (dashed lines in Fig.16) which were linearly stable in the initial linear phase became unstable in the non-linear phase. This is similar to the findings of low-n structures induced by non-linear coupling in multi-harmonic ELM modelling presented in [17]. The spectrum of temperature fluctuations in the inter-ELM period after a crash (Fig.17) showed the presence of coherent modes $n=5-8$ during a few $m s$. Note that the dominant harmonic number can change in time during the pedestal build-up in modelling, which is similar to ECEI observations [4]. The experimental ELM frequency for pulse KSTAR\#7328 was about $40 \mathrm{~Hz}$, hence inter-ELM period was $\sim 25 \mathrm{~ms}$. This is a too long-lasting inter-ELM period for the modelling, since multi-harmonics are highly consuming in terms of computing time and memory. Moreover the time step in inter-ELM period modelling should be kept of the order of a few Alfven times $\left(\sim 10^{-4} \mathrm{~ms}\right)$ to resolve all harmonics fluctuations in time. In order to achieve multi-harmonics and multi-ELM regime on a more reasonable and shorter time accessible for modelling, the heating power was artificially increased to $9 M W$ in modelling instead of $3 M W$ in experiment. At the same time the toroidal velocity was increased $(487 \mathrm{~km} / \mathrm{s}$ instead of $325 \mathrm{~km} / \mathrm{s}$ in experiment) to increase the stabilizing effect of the rotation on the remaining MHD after ELM crash which allows the pedestal to re-build on a shorter time scale similar to [15]. The $1.2 \mathrm{kHz}$ multi-ELMs were obtained using $n=8$ single 
harmonic (Fig.18). Note that for the established ELM cycles (last two ELMs in Fig.18) the pressure and current profiles before an ELM are similar (Fig.19). In multi-harmonics $n=1-8$ simulation (Fig.20) only two full ELM crashes were modelled here, since this case demands a much longer computer simulation time, that was limited. In the case of multi-harmonics the second ELM is mostly due to the $n=6$ mode compared to the first ELM where $n=8$ is dominant (Fig.20 bottom frame). The frequency spectrum of the electron temperature fluctuations in the mid-plane on LFS (Fig.20 top frame) for this case is presented in Fig.20 (middle frame), showing a $n=6$ 'precursor' prior to the second ELM lasting about $\sim 0.15 \mathrm{~ms}$. The pedestal pressure and current profiles before the first and the second ELM are different (Fig.21) which explains different the most unstable mode numbers seen in modelling ( $n=8$ for the first ELM and $n=6$ for the second one). As it was discussed in [15] the established ELM cycles can have different most unstable toroidal harmonic number compared to the first ELM in modelling, since the initial plasma profiles usually are arbitrarily set to be unstable and the initial perturbations are set at the numerical noise level which is not the case for the following ELM cycles.

Sec.V. Discussion and conclusions. The non-linear MHD modelling of the full ELM crash dynamics was done using the JOREK code with two fluid diamagnetic and neoclassical effects $[8,10]$ for the KSTAR pulse \#7328 parameters and compared to the ECEI diagnostic observations [3]. Most of the experimentally observed features were reproduced in modelling. In particular the structure and localization of the medium $n(n=5-8)$ peeling-ballooning modes in the pedestal region inside the separatrix, poloidal rotation frequencies and the direction of the modes rotation before ELM crash are similar to the experimental observations. It was shown that the observed poloidal rotation of the modes in the inter-ELM periods far from the ELM crash is of the order of the $\sim \vec{E} \times \vec{B}$ velocity and can be in the electron diamagnetic direction (more common observation in many tokamaks [2-7]) and in the ion diamagnetic 
direction at relatively large toroidal rotation, which was the case for the KSTAR [3-4] pulse modeled in the present paper. On the highly non-linear phase of ELM crash the regular rotation of the modes decreases and ELM filaments are expelled to the SOL. More ELM power is found in the inner divertor (in/out =2:1) compared to the outer divertor with two fluid diamagnetic and $\vec{E} \times \vec{B}$ drifts included in the model [15] which is similar to the experimental findings [16]. Multi-modes $(n=1-8)$ modelling demonstrated the acceleration of the growth of all peeling-ballooning modes and the destabilization of the previously linearly stable modes while approaching the ELM crash. This is due to the strong non-linear coupling of the modes in this phase as it was also found in [17]. In multi-ELMs regimes in the interELM periods and before the ELM crash the temperature fluctuations spectrum in modelling is similar to the one observed in experiment. In particular the presence of several unstable modes $(n=5-8)$ in the range of frequencies $(5-30 \mathrm{kHz})$ were obtained. The time duration of these coherent structures varies from $0.15 \mathrm{~ms}$ to $2 \mathrm{~ms}$ in modelling. Note however that this work represents only a first step in the interpretation of the experimental observations of ELM precursors and obviously has its limits. In the first place the exact pedestal measurements were not available on KSTAR, so there was a certain freedom in the choice of the pedestal pressure and its gradient which defines value of the diamagnetic velocity. The resistivity was two orders higher in modelling than in experiment for numerical stability reasons. At the realistic resistivity the linear growth rate of the modes will probably change to smaller values, but the general dynamics will remain. The resistivity scans within possible from the numerical point of view range confirms this statement. Limitations in the computer time and memory for multi-harmonics simulations did not permit to achieve realistic experimental ELM frequency, however it could be artificially increased in modelling without changing much the generic picture of multi-cycling ELMs behavior. The aim of this work was mainly to propose a minimum model for the possible underlying mechanism of the observed rotating 
structures in the pedestal temperature before an ELM crash (ELM precursors) and in the interELM periods providing more detailed physics basis for the understanding of ELMs.

Ackowledgement: This work has been carried out within the framework of the EUROfusion Consortium and has received funding from grant AWP15-ENR-01/IPP-05. This work was supported by the Energy oriented Centre of Excellence (EoCoE), grant agreement number 676629, funded within the Horizon2020 framework of the European Union. A part of this work was carried out using the HELIOS supercomputer system (IFERC-CSC), Aomori, Japan, under the Broader Approach collaboration, implemented by Fusion for Energy and JAEA. The part of this work was carried out using MARENOSTRUM supercomputer system (Barcelona, Spain) under PRACE project (2016) and using HPC CURIE (TGCC-Bruyères-leChâtel, France) under GENCI project(2016). The views and opinions expressed herein do not necessarily reflect those of the European Commission or ITER.

\section{References:}

[1] A Loarte et al Plasma Phys. Contr. Fusion 45, 1549 (2003)

[2] G Yun et al Phys Rev Lett 107,045004(2011)

[3] M. Kim et al Nucl. Fusion 54, 093004 (2014)

[4] J E Lee et al Nucl Fusion 55, 113035(2015)

[5] I Classen et al Nucl Fusion 52,1223009 (2012)

[6] A Kirk et al Nucl Fusion 54,114012(2013)

[7] Y Sechrest et al Nucl Fusion 52,123009(2012)

[8] G T A Huysmans et al Nucl. Fusion 47,659 (2007)

[9] J Morales Phys of Plasmas 23,042513(2016)

[10] F Orain et al Phys of Plasmas 20, 102510 (2013)

[11] M Becoulet Nucl. Fusion 52 (2012) 054003

[12] O Czarny et al. J.Comp.Phys. 227, 2008.

[13] P. Henon et al. Parallel Comp. 34 345-362, 2008.

[14] G Huysmans Plasma Phys Control Fusion 47 B165(2005)

[15] F Orain et al Plasma Phys Control Fusion, 57, 014020(2015)

[16] R A Pitts et al Nucl. Fusion 47 (2007) 1437-1448 
[17] I Krebs et al Physics of Plasmas, 20, 082506 (2013)

\section{Figure captions:}

Fig.1. Left-handed coordinate system $\left(\vec{e}_{r}, \vec{e}_{\theta}, \vec{e}_{\varphi}\right)$ used for the analysis of ballooning modes rotation.

Fig.2. Initial density (dashed black), temperature (blue), pressure (cross magenta) profiles used in JOREK modelling.

Fig.3. Toroidal rotation profile used in modelling (blue)and measured by CES diagnostic (red squares).

Fig.4. Two ECEI diagnostic images of temperature fluctuations before ELM crash separated by $\sim 0.008 m s$ in time.

Fig.5. The time evolution of the magnetic energy of the single harmonic $n=8$ during full ELM crash calculated by JOREK.

Fig.6. Two images of temperature fluctuations before ELM crash separated by $\sim 0.008 \mathrm{~ms}$ in time in non-linear MHD modelling in single harmonic $n=8$ simulation at Vtor $=325 \mathrm{~km} / \mathrm{s}$.

Fig.7. Two images of temperature fluctuations before ELM crash separated by $\sim 0.008 m s$ in time in JOREK modelling in single harmonic $\mathrm{n}=8$ simulation at $V t o r=54 \mathrm{~km} / \mathrm{s}$.

Fig.8. The profiles of the velocities in mid-plane : $V_{E \times B}$ (magenta solid line for Vtor $=325 \mathrm{~km} / \mathrm{s}$ and magenta dashed for Vtor $=54 \mathrm{~km} / \mathrm{s}) V_{\theta \text {,mode }}^{f}=0.5 V_{i}^{*}$ (red bold line) ;

$V_{\theta \text {,mode, }, a b}=V_{E \times B}+V_{\theta \text {,mode }}^{f}$ ( black crosses on the solid line for Vtor $=325 \mathrm{~km} / \mathrm{s}$ and black crosses on dashed line for Vtor $=54 \mathrm{~km} / \mathrm{s}$ )

Fig.9. Edge magnetic topology at a time corresponding to a maximum of magnetic energy of $n=8$ ballooning mode (indicated by star in Fig.5)

Fig.10. Density (upper scale in $\left[10^{20} \mathrm{~m}^{-3}\right]$ ) filaments during non-linear phase of an ELM and maximum parallel heat flux (bottom scale in [GW/m2]) in the inner and outer divertor due to an ELM. 
Fig.11. Snapshots of temperature fluctuations in the mid-plane on LFS before and during ELM crash. Images are separated in time by $\sim 0.0166 \mathrm{~ms}$.

Fig.12. Poloidal plasma velocity profile at mid-plane on LFS. Before an ELM (time of the frame 1 in Fig.11)-in bold, During non-linear phase (time corresponds to the frame 4 in Fig.11) -in dashed.

Fig.13. Poloidal plasma velocity at mid-plane on LFS. Before an ELM (top figure) and at maximum magnetic energy of $n=8$ mode during ELM crash (bottom).

Fig.14. Density filaments and blobs during non-linear phase of ELM n=8 . Electrostatic potential contours are superposed on the plot indicating ExB convective cells formation during an ELM.

Fig.15. Relaxation of density (in red) and temperature (in blue) profiles during an ELM (modelling with single harmonic $n=8$ ). Profiles before ELM crash (with markers) correspond to the frame 1 and after crash (plain lines) to the frame 6 in Fig.11.

Fig.16. Magnetic energy in multi-harmonics $(n=1-8)$ simulation of ELM in KSTAR. Dashed lines indicate initially linearly stable, but then non-linearly unstable modes $(n=1-3)$.

Fig.17. Temperature fluctuations in the pedestal at mid-plane in JOREK modelling (upper frame), frequency spectrum (middle), magnetic energy in time (bottom) for multi-harmonics $n=1-8$ simulation of an ELM on KSTAR for Vtor=325km $/ \mathrm{s}$.

Fig.18. Magnetic energy versus time for multi-cycles simulations for single harmonic $n=8$, Vtor $=487 \mathrm{~km} / \mathrm{s}$, NBI power was taken $9 \mathrm{MW}$.

Fig.19. Pedestal pressure and current profiles in mid-plane at LFS before the last two established single harmonic $n=8$ ELMs presented in Fig.18.

Fig.20. Temperature fluctuations in the pedestal at mid-plane in JOREK modelling (upper frame), frequency spectrum (middle) and evolution of the magnetic energy in time (bottom) 
for multi-harmonics simulation $(\mathrm{n}=1-8)$ of an ELM at increased Vtor $=487 \mathrm{~km} / \mathrm{s}$ and $9 M W$ NBI power.

Fig.21. Pedestal pressure and current profiles in mid-plane at LFS before the first (most unstable mode was $n=8$ ) and second (most unstable was $n=6$ ) ELM in multi-harmonics $n=1-8$ simulation presented in Fig.20.

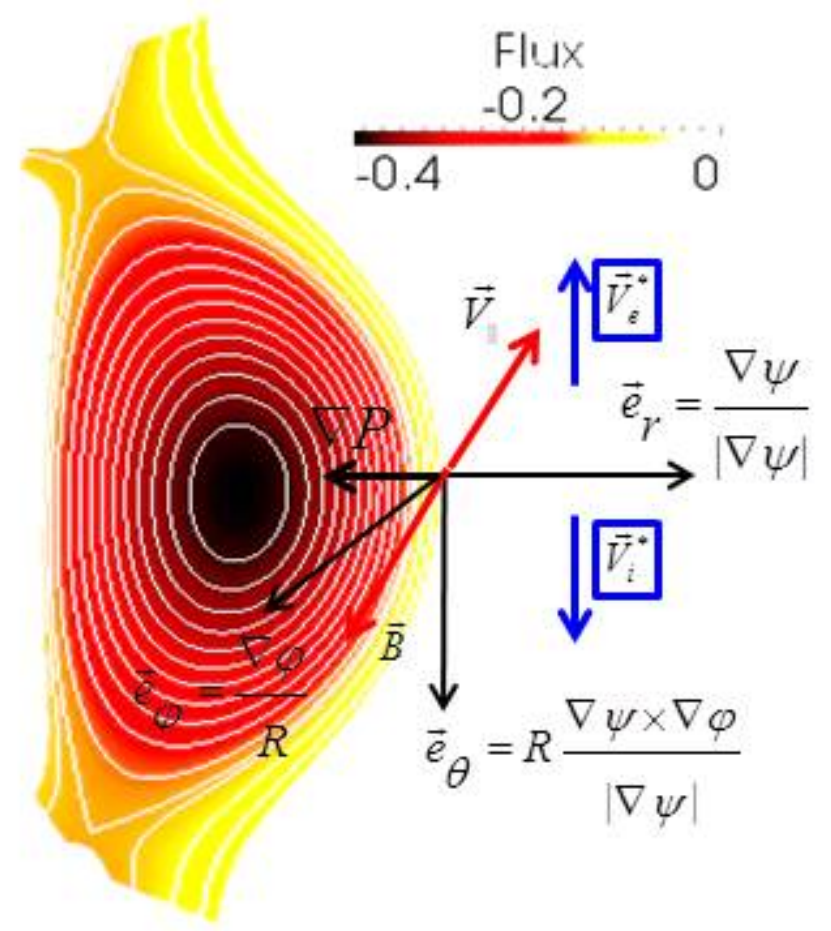

Fig.1 


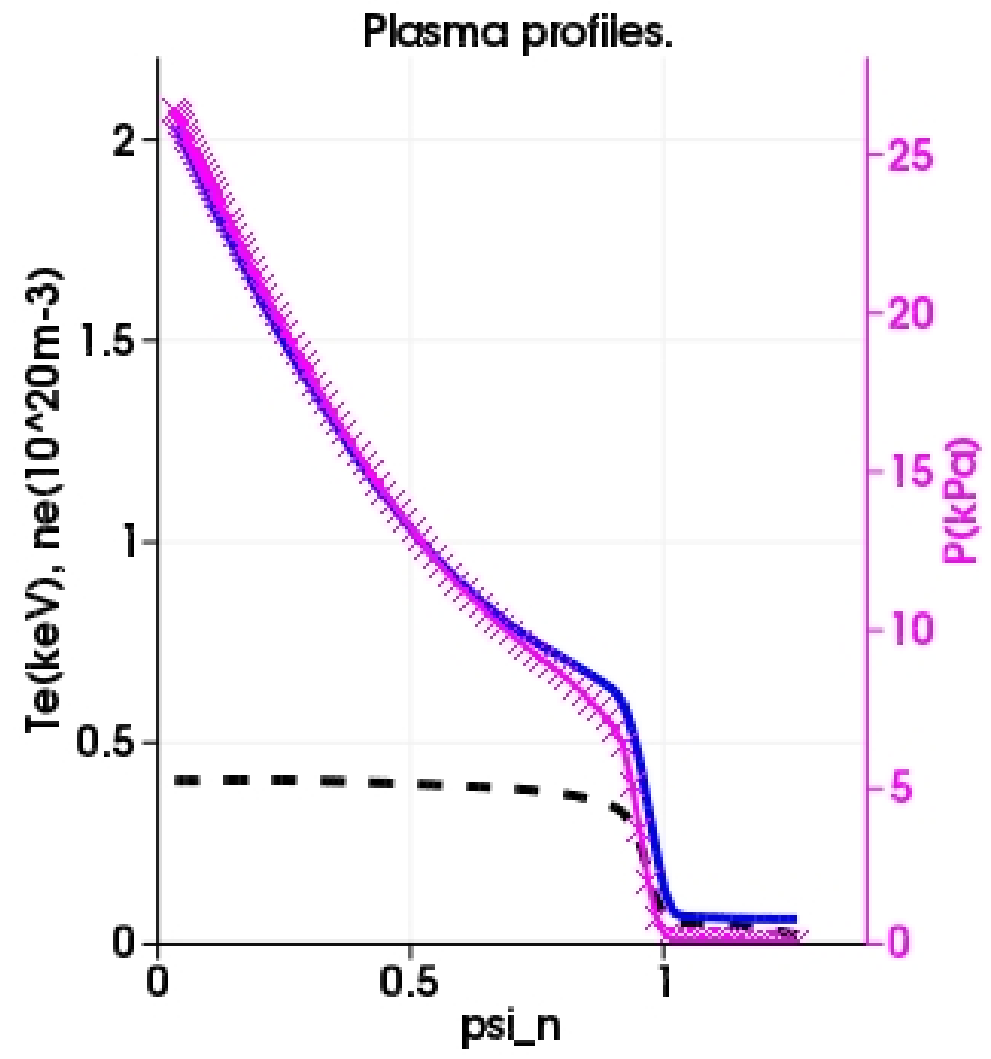

Fig.2

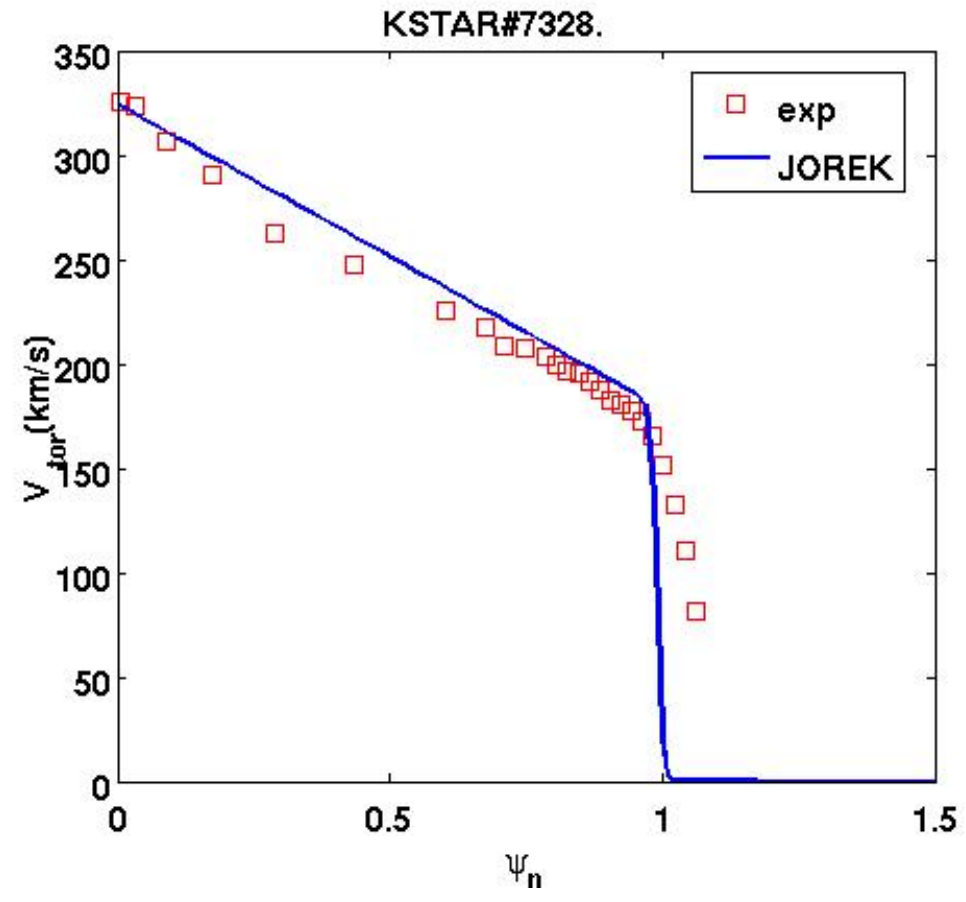

Fig.3 


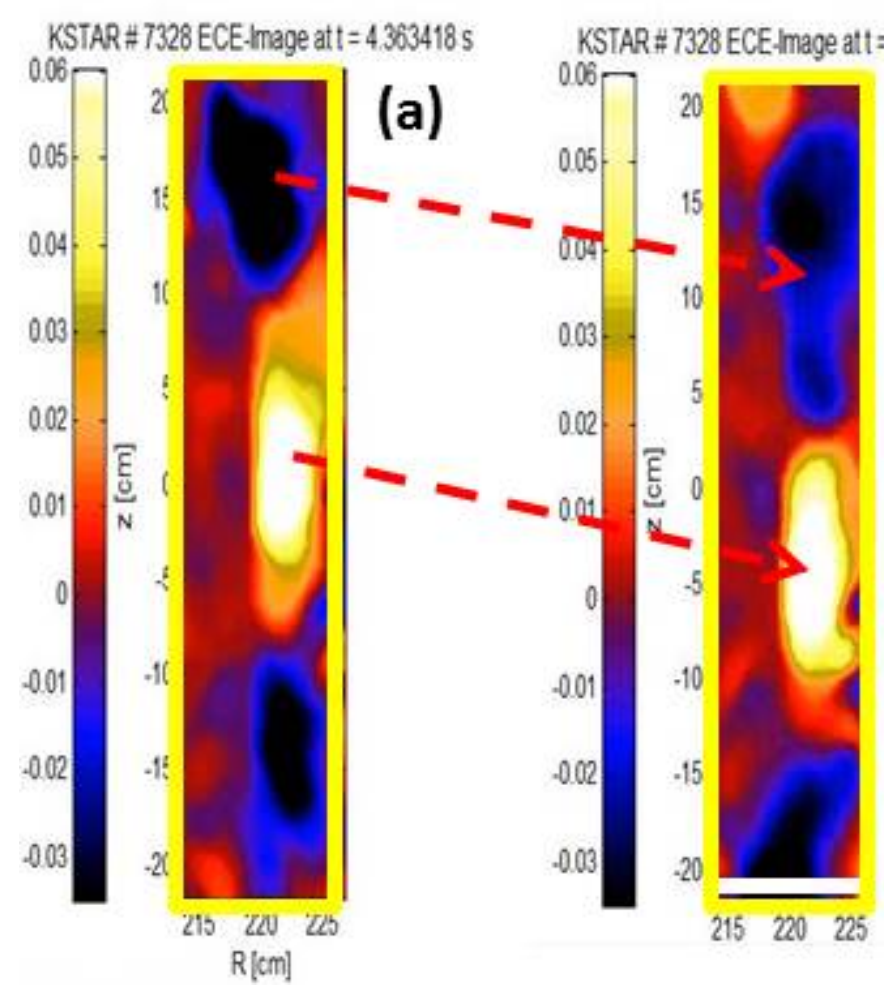

(b)

Fig.4

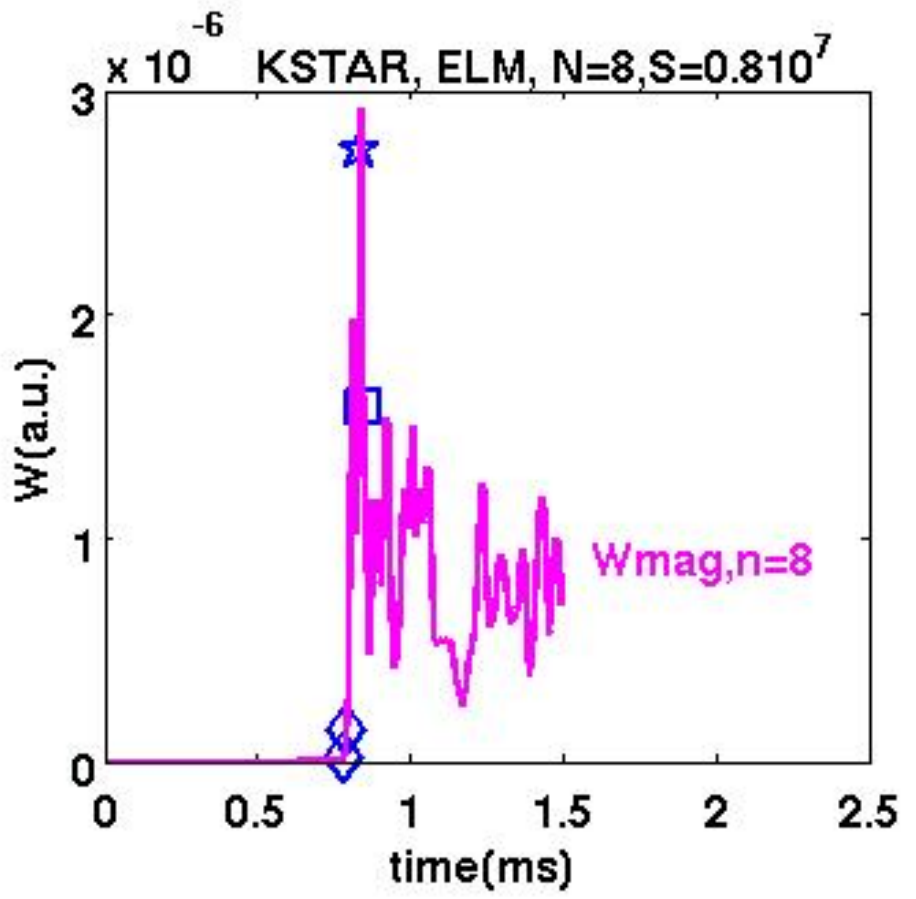

Fig.5 


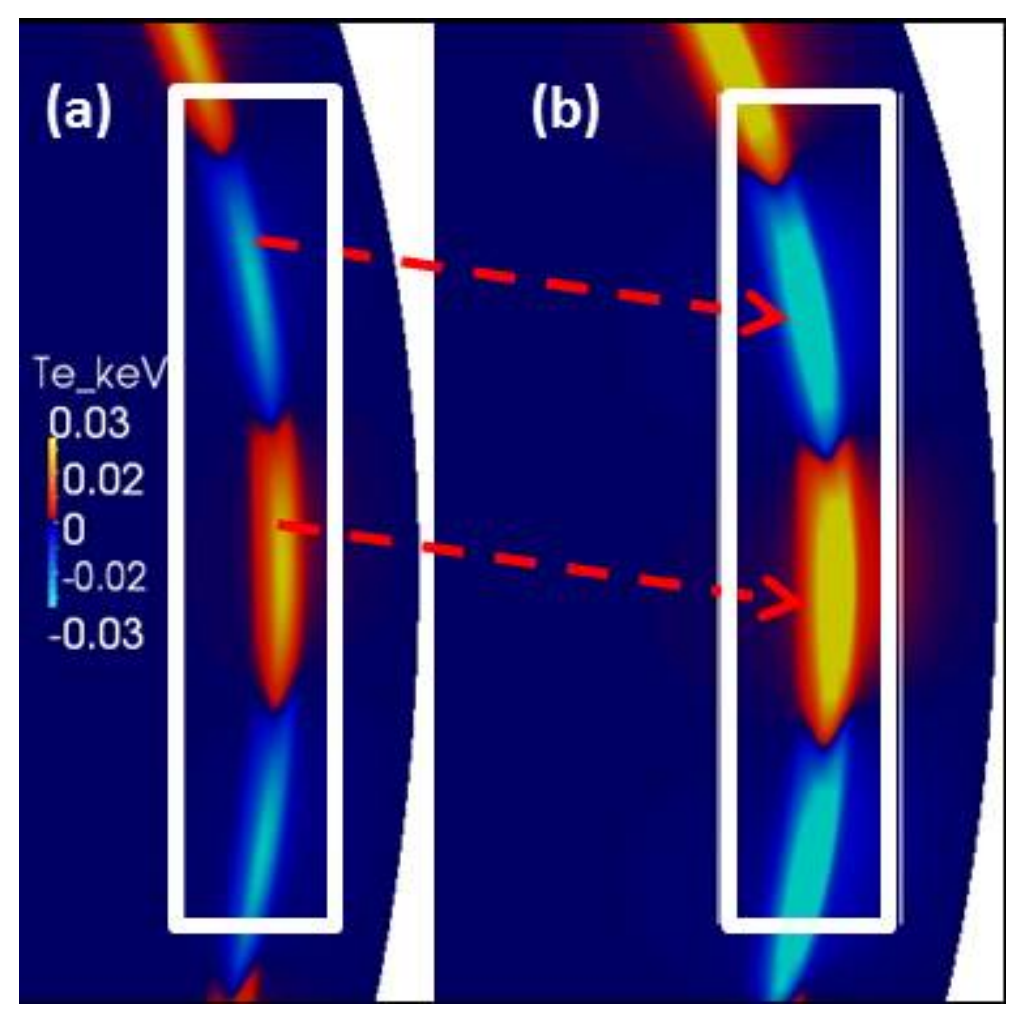

Fig.6

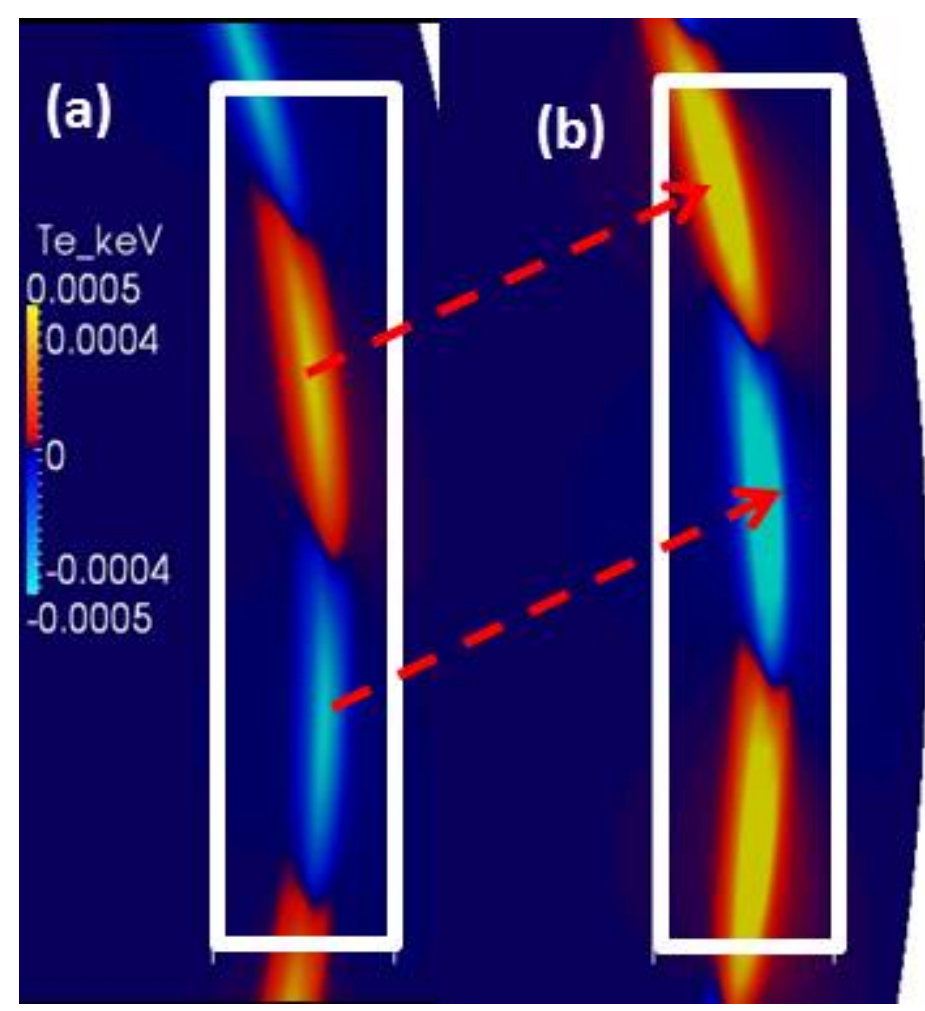

Fig.7 


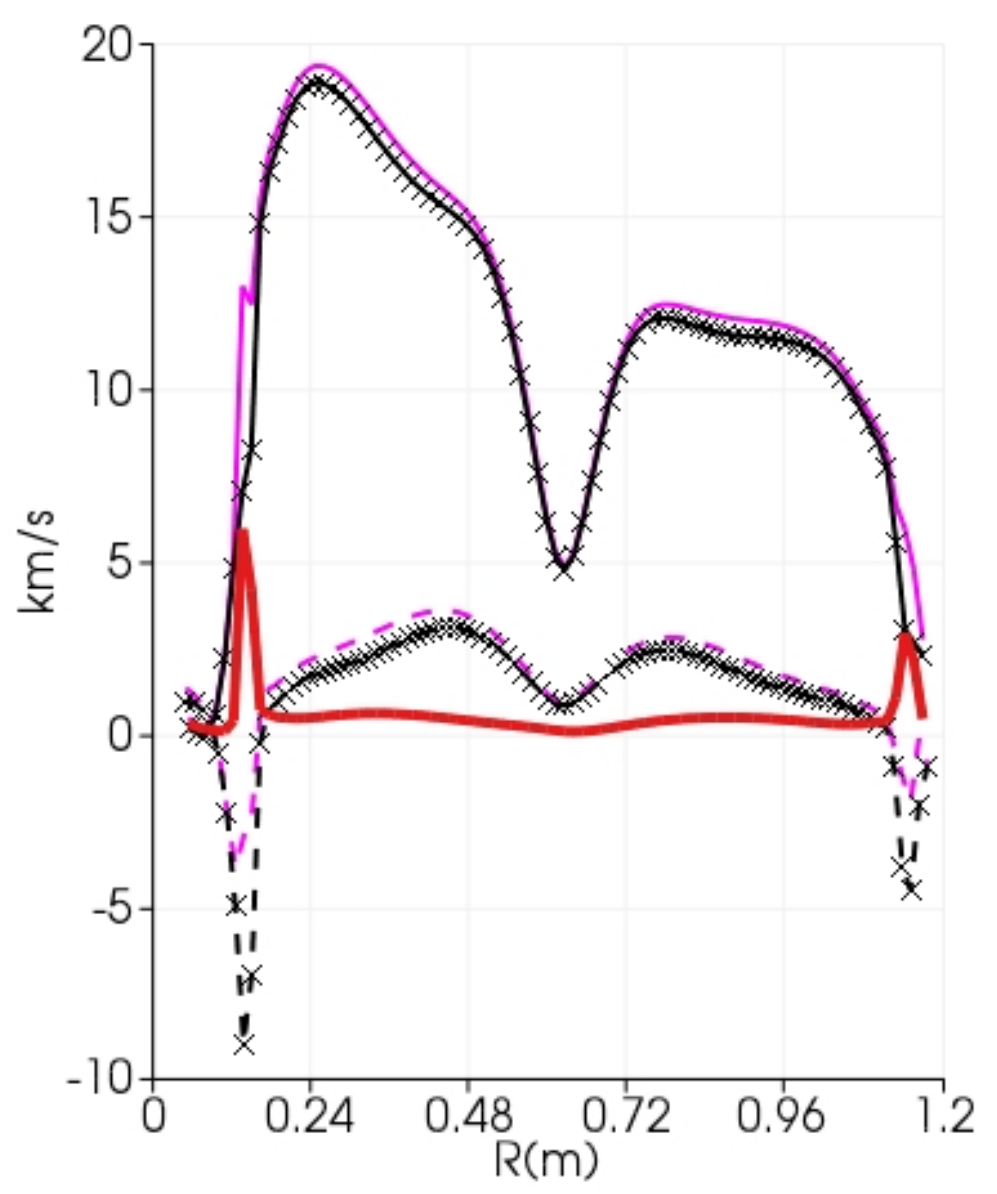

Fig.8

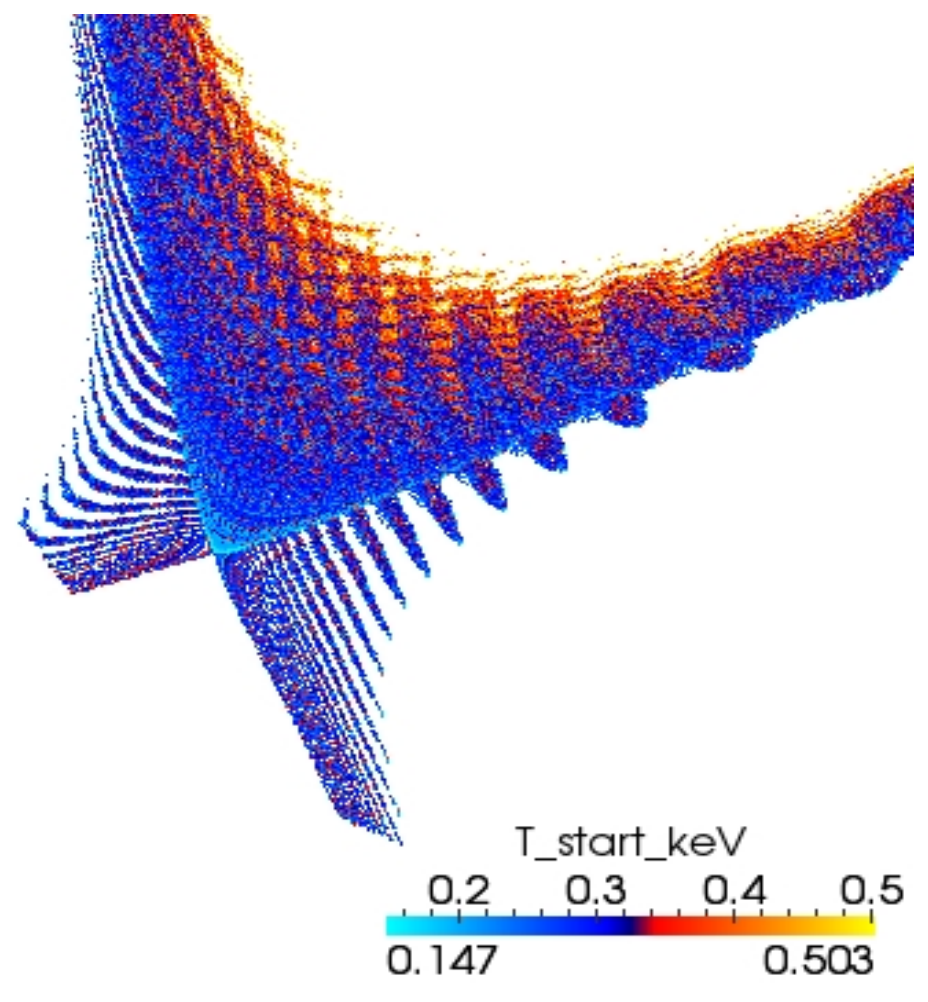

Fig.9 


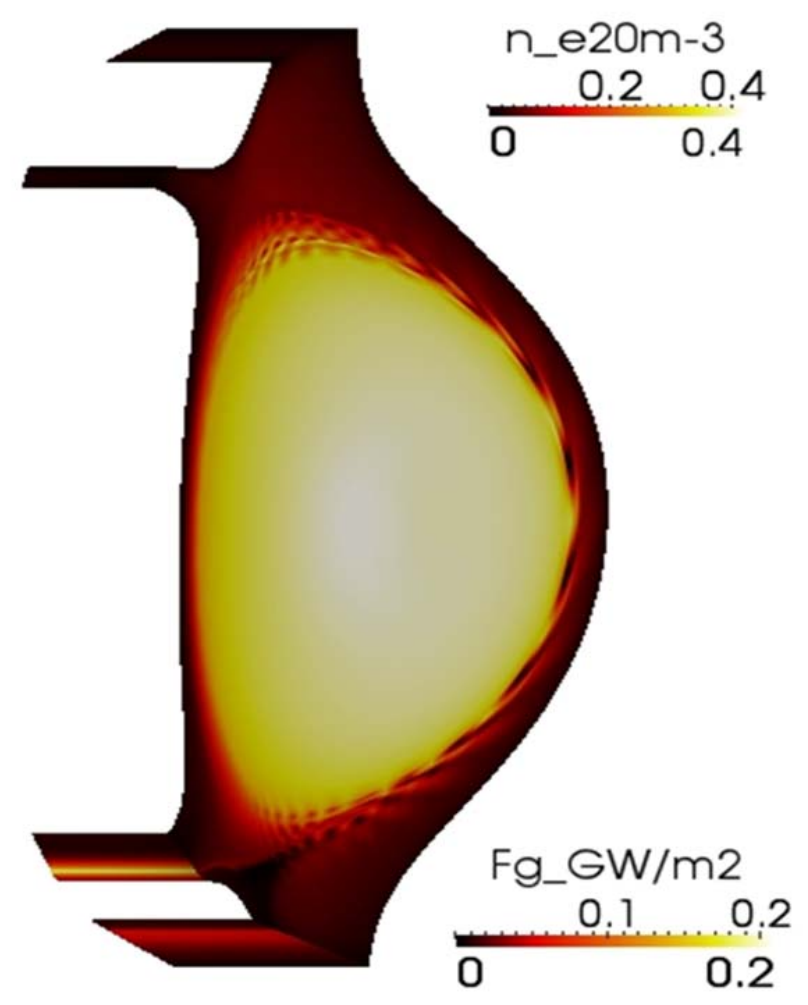

Fig.10
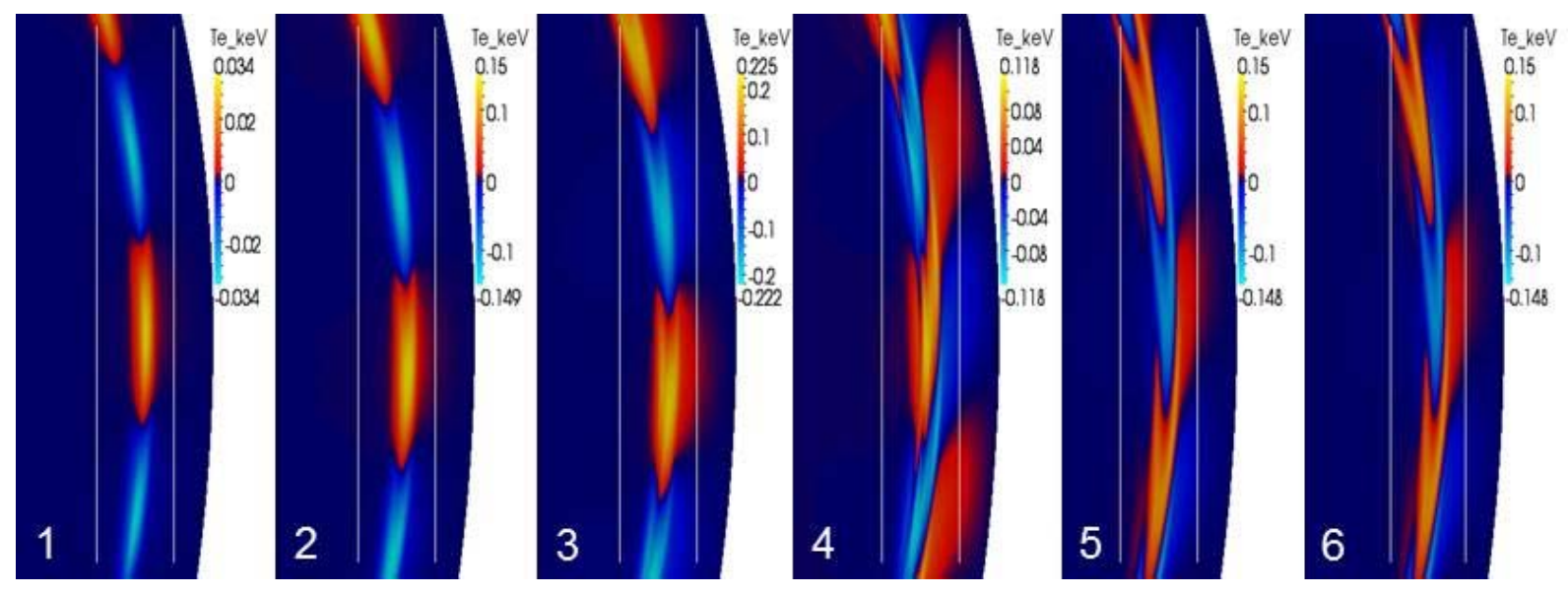

Fig.11 


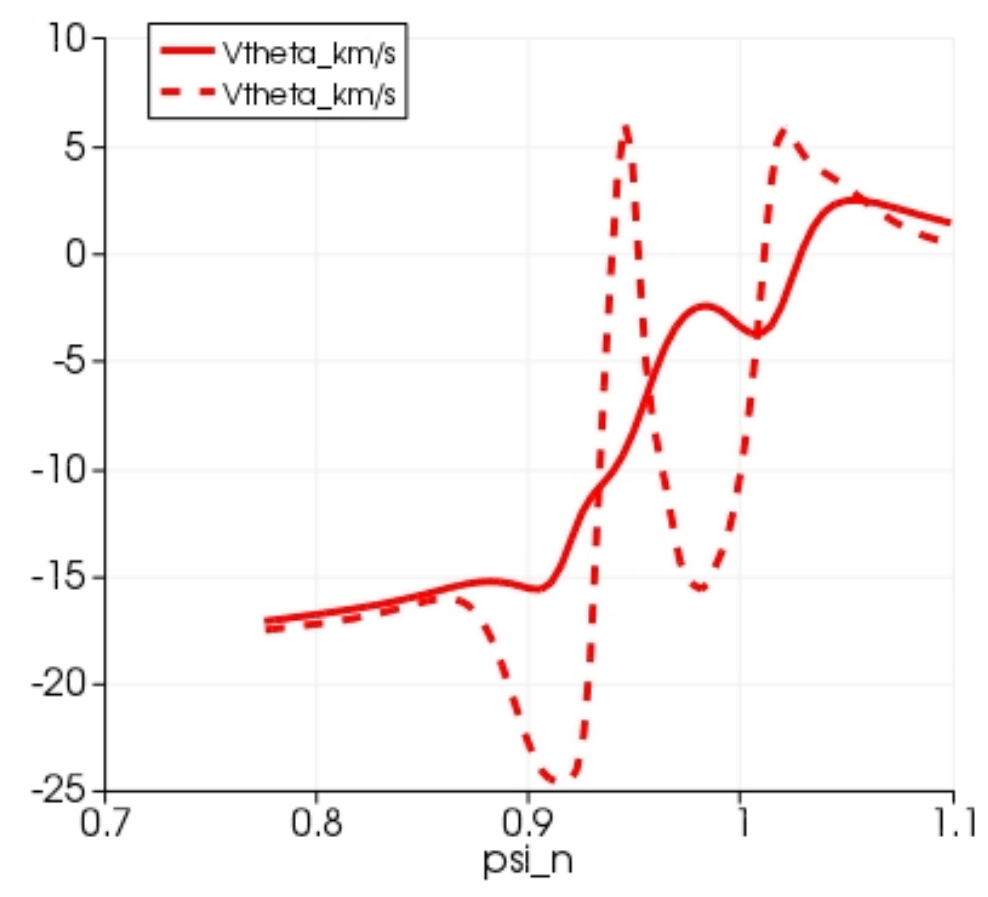

Fig.12
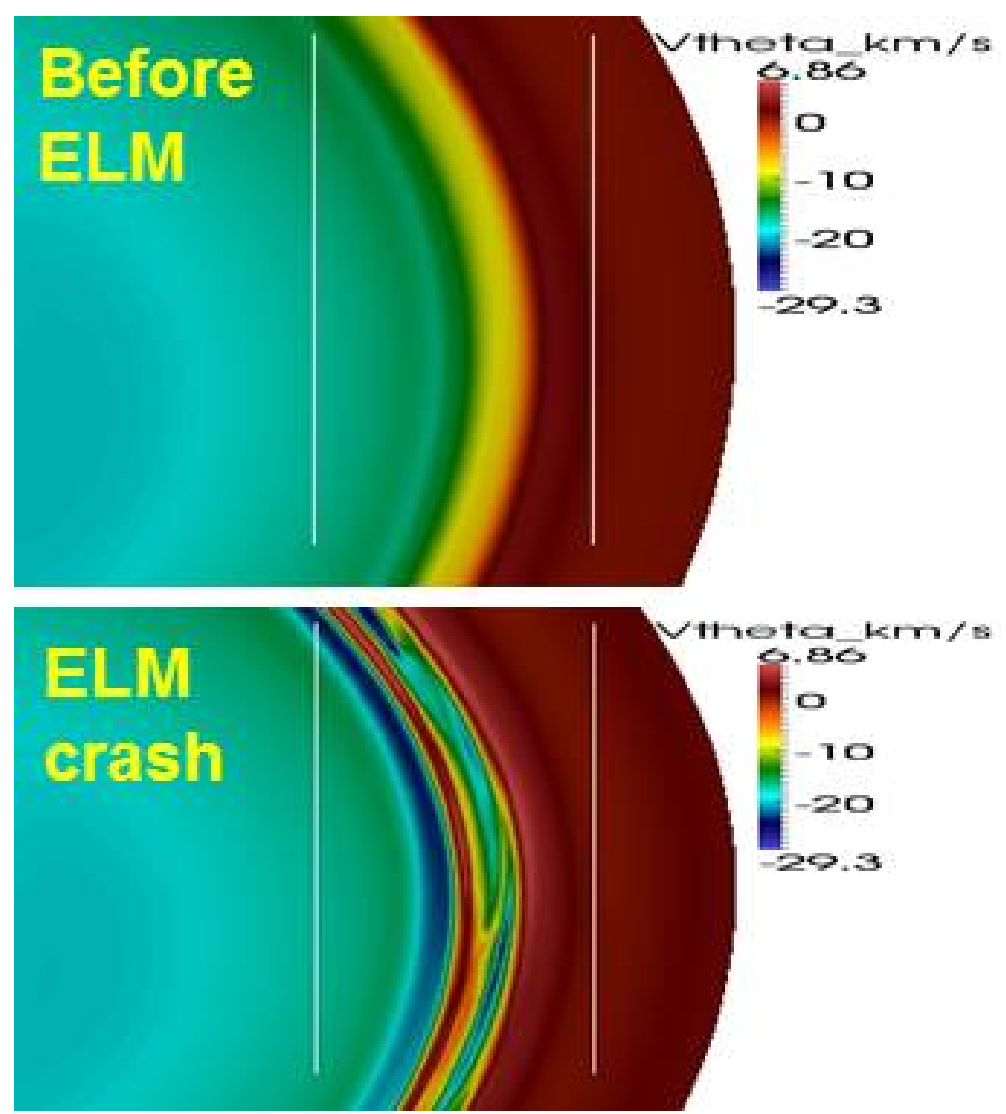

Fig.13 


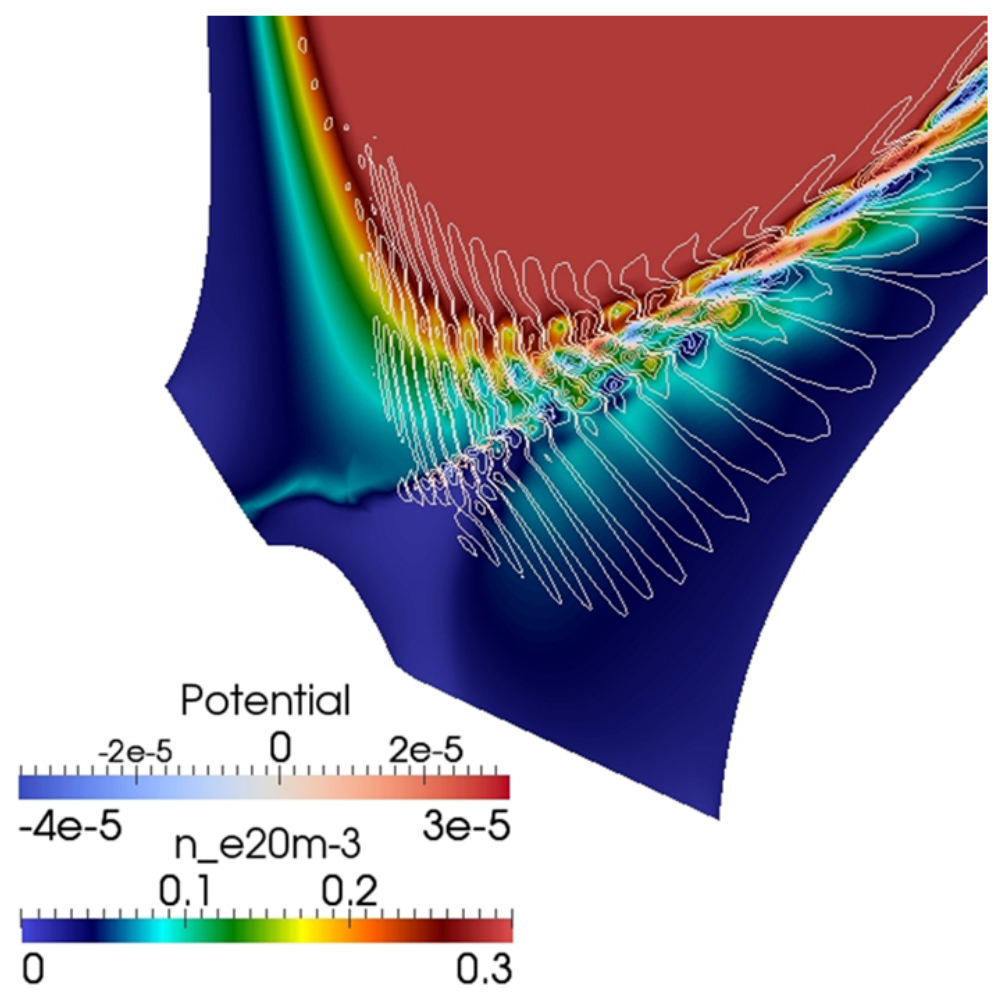

Fig.14

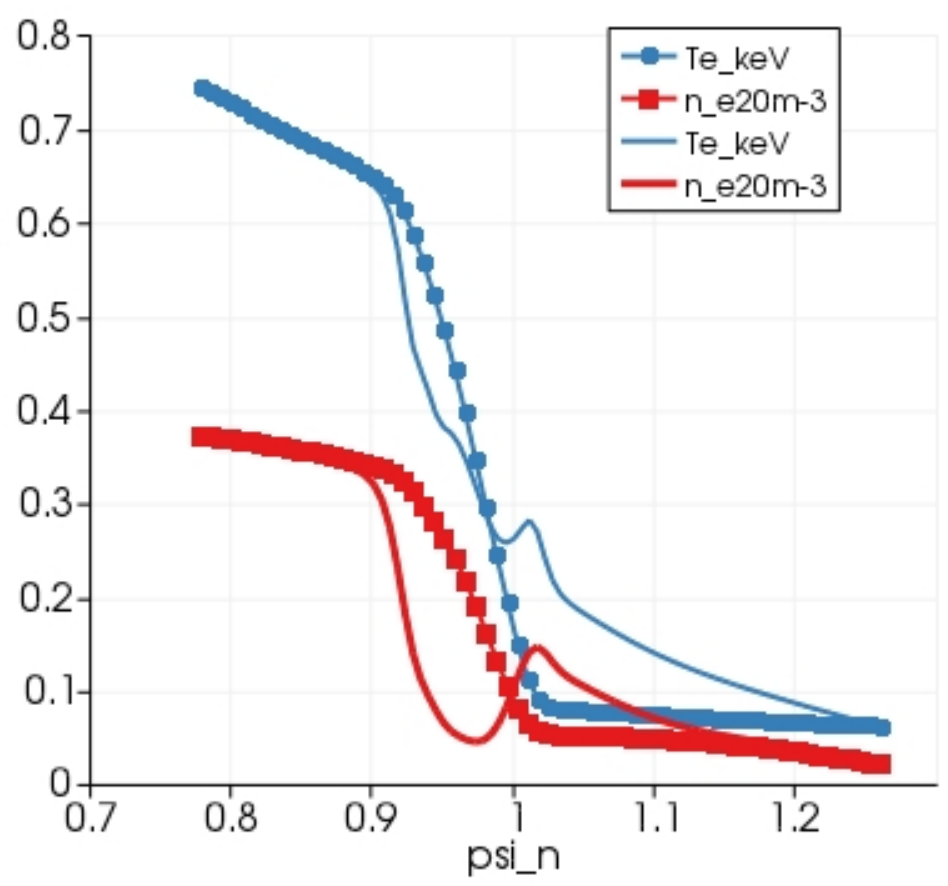

Fig.15 


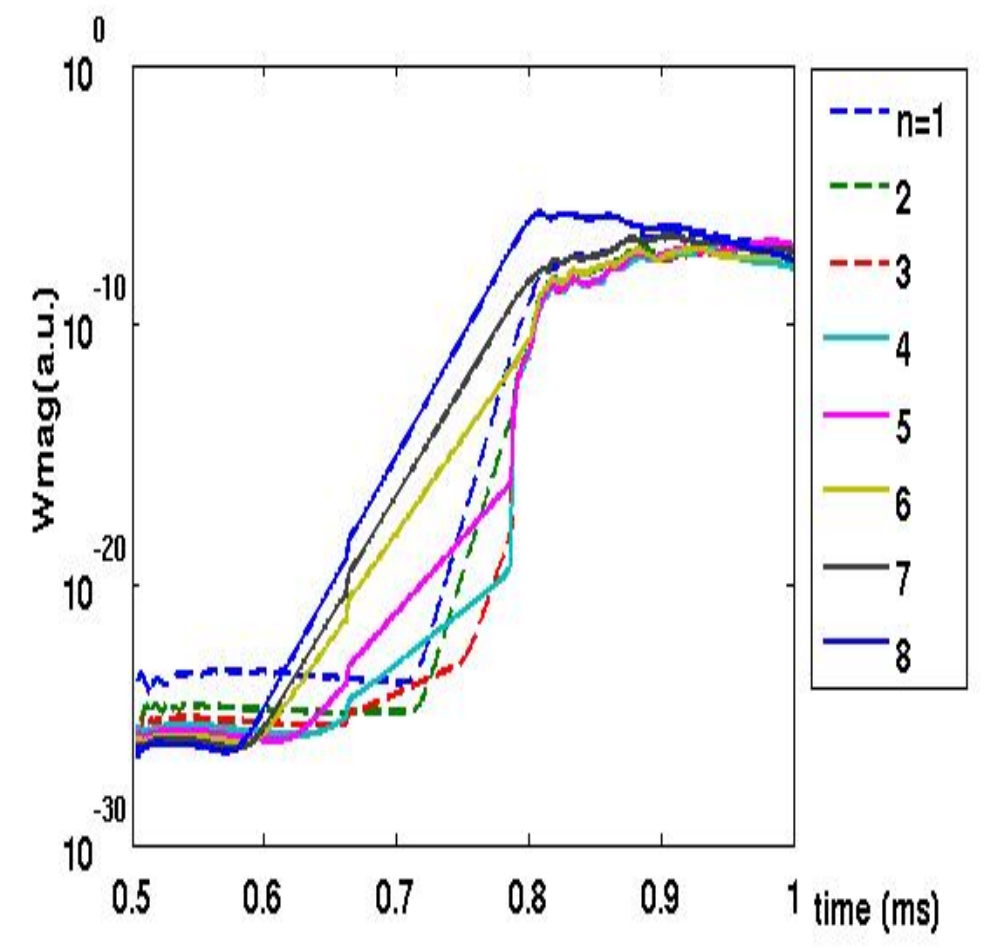

Fig.16

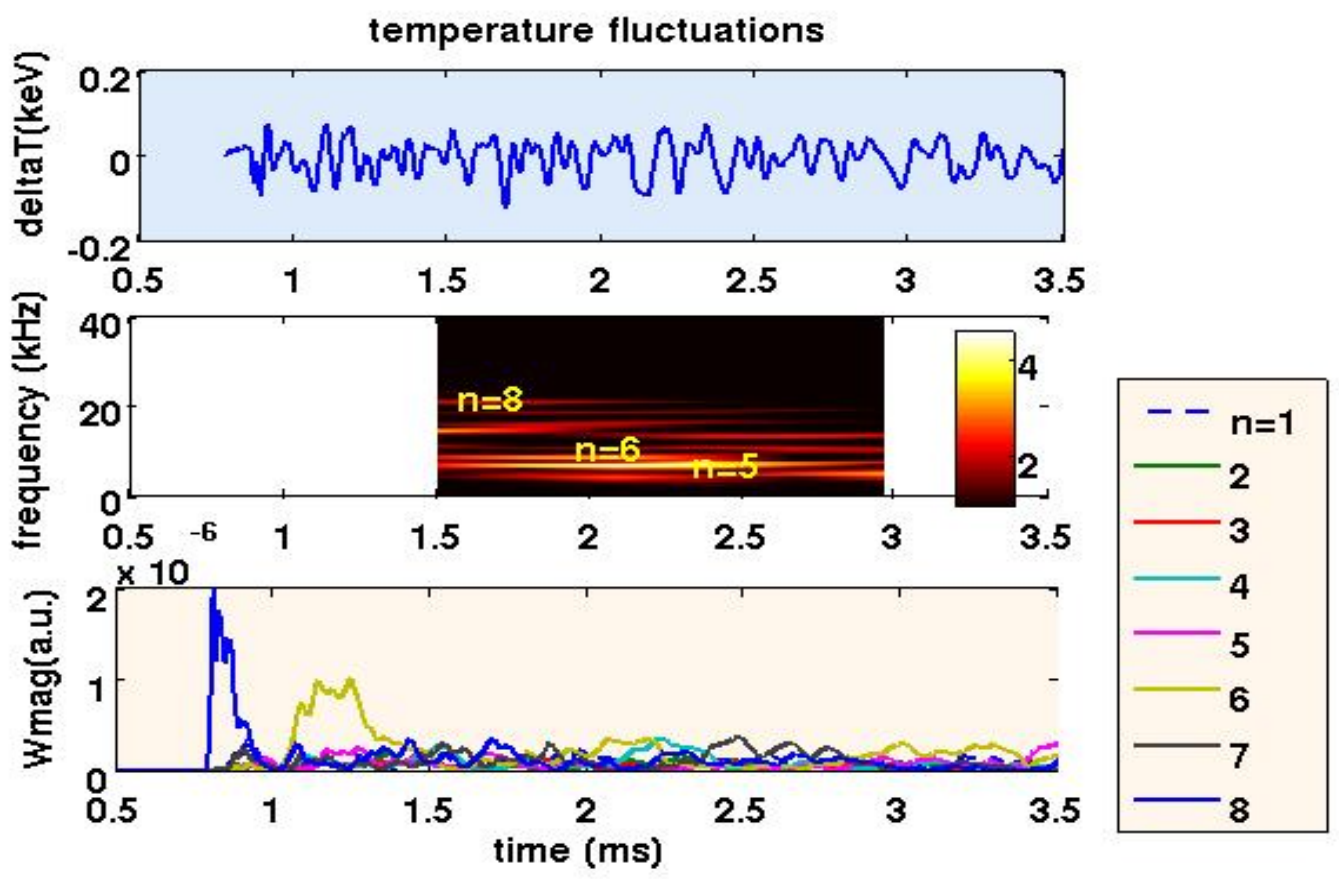

Fig.17 


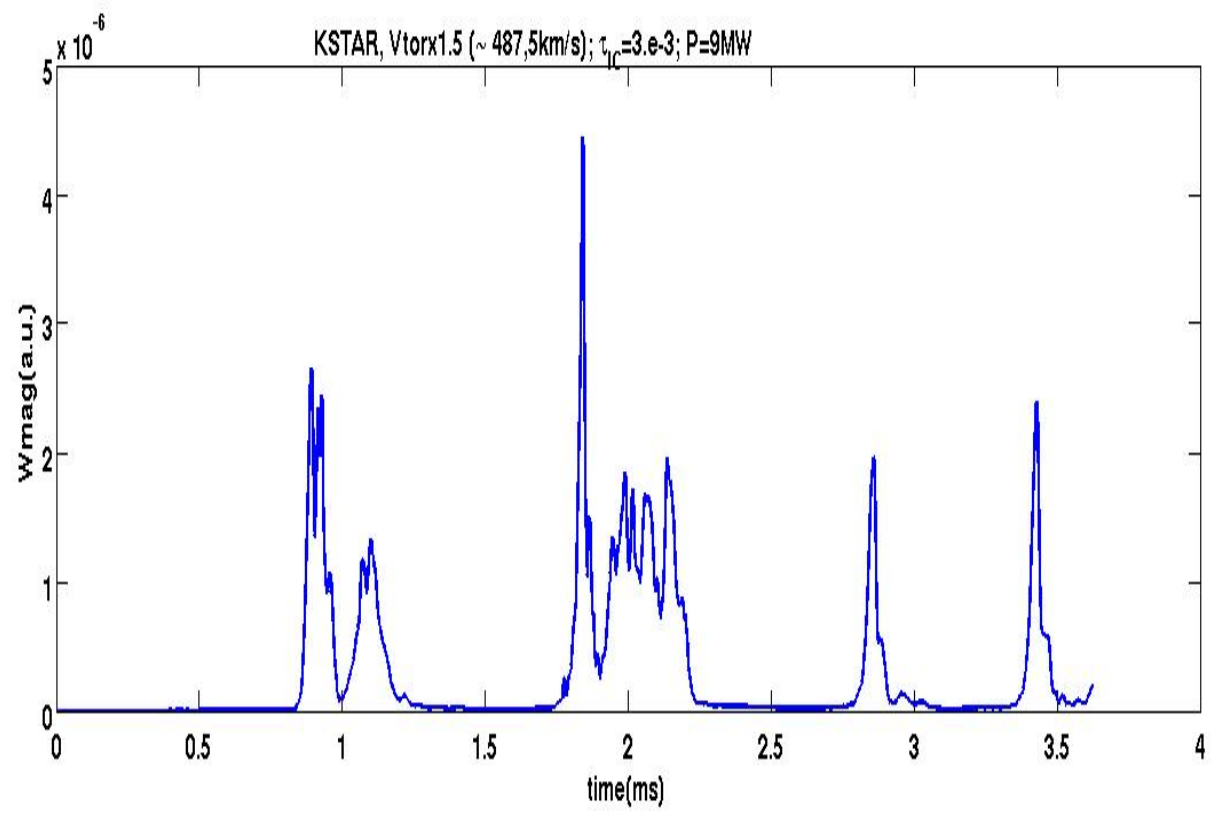

Fig.18

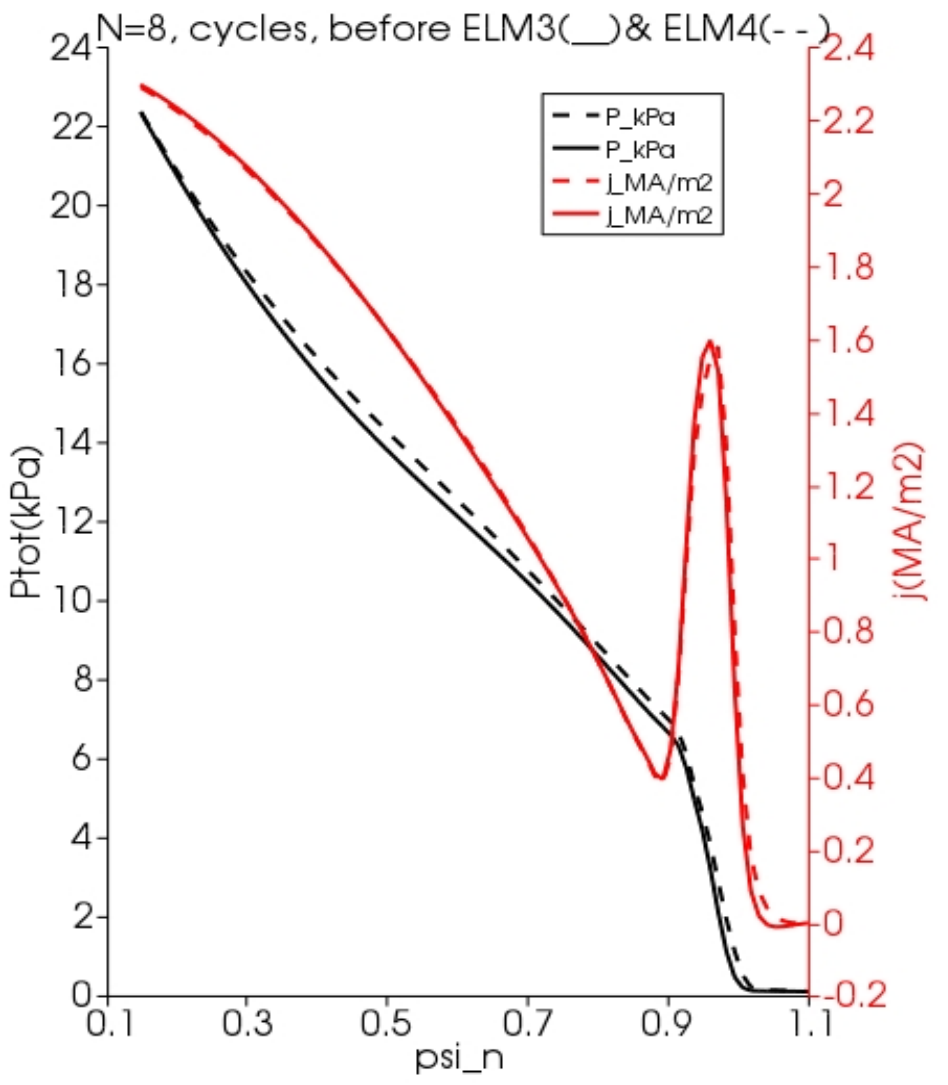

Fig.19 


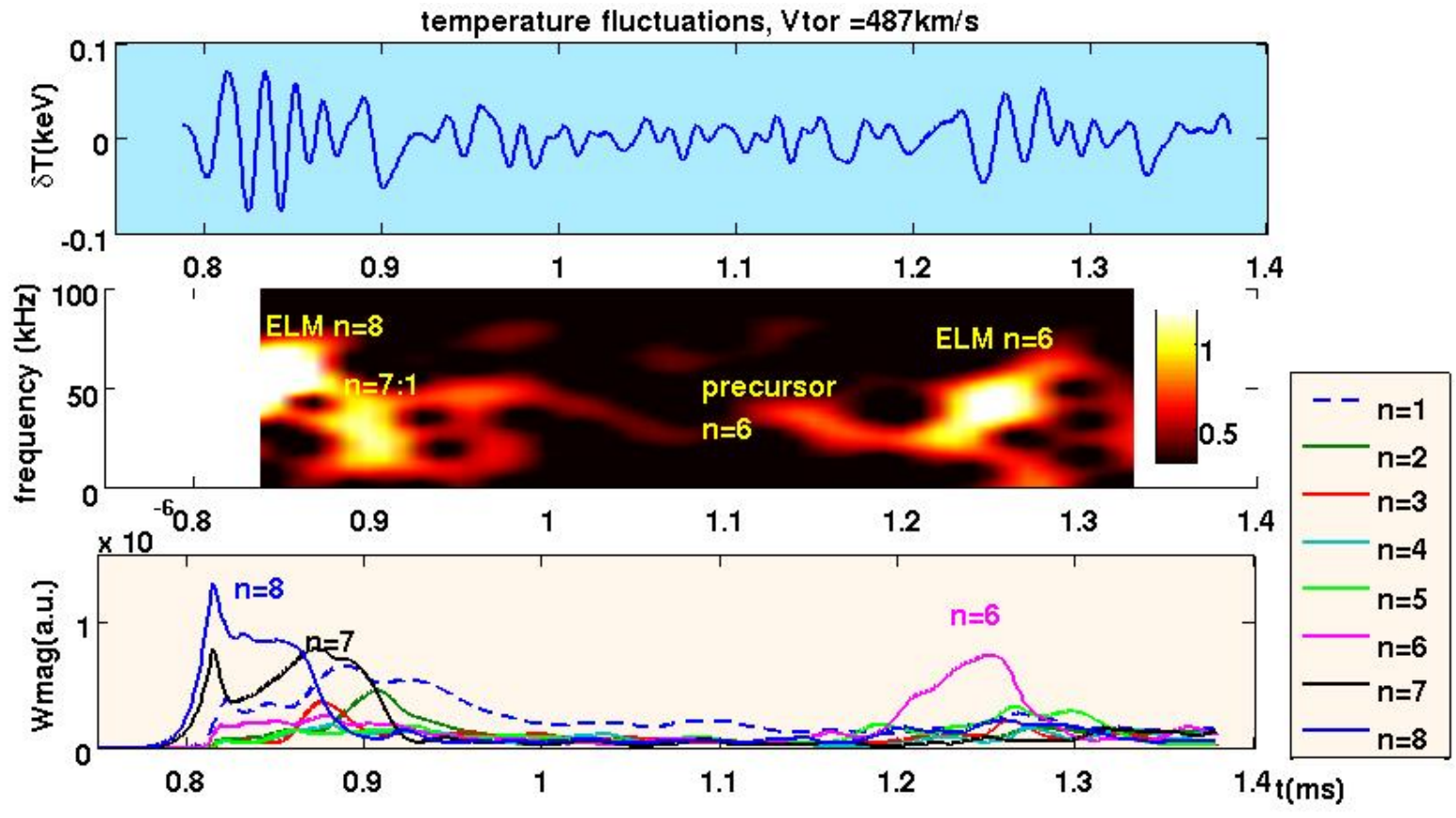

Fig.20

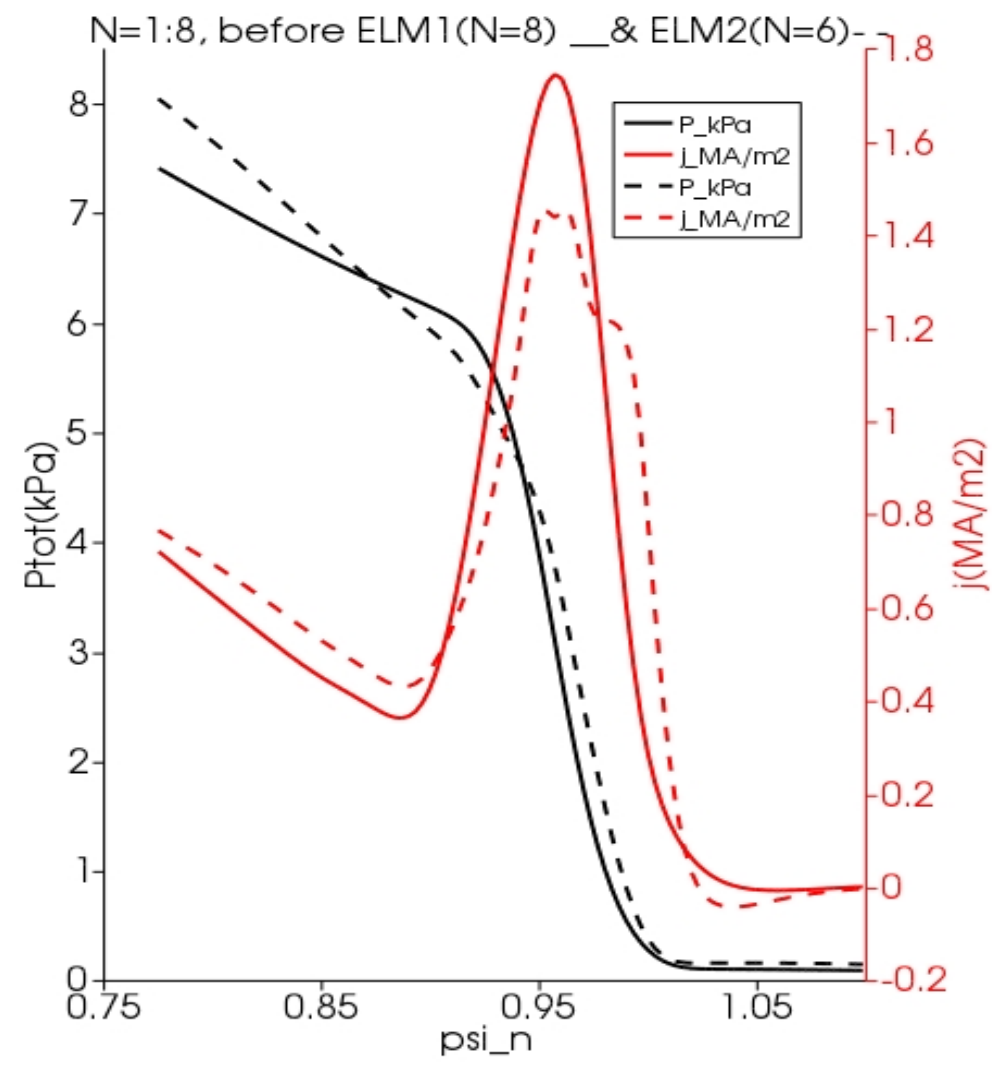

Fig.21 